THE ASTROPHYSICAL JOURNAL, 560:749-762, 2001 October 20

(C) 2001. The American Astronomical Society. All rights reserved. Printed in U.S.A.

\title{
HIGH SPECTRAL AND SPATIAL RESOLUTION OBSERVATIONS OF SHOCKED MOLECULAR HYDROGEN AT THE GALACTIC CENTER
}

\author{
F. YUSEF-ZADEH \\ Department of Physics and Astronomy, Northwestern University, Evanston, IL 60208; zadeh@northwestern.edu \\ S. R. STOLOVY \\ SIRTF Science Center, Caltech, Pasadena, CA 91125; stolovy@ipac.caltech.edu
}

\section{BURTON}

School of Physics, University of New South Wales, Sydney, NSW 2052, Australia; and School of Cosmic Physics, Dublin Institute for Advanced Studies, 5 Merrion Square, Dublin 2, Ireland; M.Burton@unsw.edu.au

M. WARDLE

School of Physics, University of Sydney, NSW 2006, Australia; wardle@physics.usyd.edu.au

\section{AND}

M. C. B. Ashley

School of Physics, University of New South Wales, Sydney, NSW 2052, Australia; M.Ashley@unsw.edu.au Received 2001 March 21; accepted 2001 June 12

\begin{abstract}
The presence of $\mathrm{OH}(1720 \mathrm{MHz})$ masers and the absence of counterparts at $1665 / 1667 \mathrm{MHz}$ has proved to be a clear diagnostic of shocked molecular gas associated with Galactic supernova remnants. This suggests that shocked molecular gas should be associated with the $\mathrm{OH}(1720 \mathrm{MHz})$ masers that have been detected in the circumnuclear disk (CND) and Sgr A East at the Galactic center. In order to test this hypothesis, we observed the $\mathrm{H}_{2} 1_{-0} S(1)$ and $\mathrm{Br} \gamma$ lines using NICMOS on the Hubble Space Telescope (HST) and the University of New South Wales Infrared Fabry-Perot (UNSWIRF) etalon at the Anglo-Australian Telescope (AAT), near the regions where $\mathrm{OH}(1720 \mathrm{MHz})$ masers are detected in the CND and Sgr A East. We present the distribution of $\mathrm{H}_{2}$ in the north and south lobes of the CND and in Sgr A East. $\mathrm{H}_{2}$ emission accompanies almost all the maser spots detected at the Galactic center. In particular, we find a striking filamentary structure near the northwest of the CND and evidence that shocked molecular gas is associated with the $70 \mathrm{~km} \mathrm{~s}^{-1}$ molecular cloud at the Galactic center. We argue that the emission from the CND could arise in gas heated by the dissipation of the random motion of clumps by collisions or the dissipation of turbulence in a more homogeneous medium. In addition, highly redshifted gas of up to $140 \mathrm{~km} \mathrm{~s}^{-1}$ close to the eastern edge of the Sgr A East shell is detected. These observations combined with $\mathrm{OH}(1720 \mathrm{MHz})$ results suggest that the $\mathrm{H}_{2}$ gas is shocked and accelerated by the expansion of Sgr A East into the 50 and the $70 \mathrm{~km} \mathrm{~s}^{-1}$ clouds and into the lobes of the CND.

Subject headings: galaxies: ISM - Galaxy: center — infrared: ISM -

ISM: individual (Sagittarius A*) — ISM: molecules — masers
\end{abstract}

\section{INTRODUCTION}

The Galactic center provides a unique opportunity to study in detail the dynamics and physical conditions of the closest galactic nucleus. The picture that has emerged from multiwavelength studies of the Galactic center over the last quarter of a century is that it contains a clumpy molecular ring (also known as the circumnuclear disk, or CND), as seen in HCN emission, on a scale of $2-5 \mathrm{pc}$, circling the Galactic center with a rotational velocity of about $100 \mathrm{~km}$ $\mathrm{s}^{-1}$ (see Jackson et al. 1993; Latvakoski et al. 1999, and references therein). The CND is heated by UV radiation from hot stars within the central cavity. The peaks of molecular emission from the CND are located in its northeast (north lobe) and southwest (south lobe) and are consistent with limb-brightening of the inner edges along the principal axis of a ring. Within the ring's central cavity, three "arms" of ionized gas (Sgr A West) are in orbital motion around the center (e.g., Roberts \& Goss 1993; Serabyn \& Lacy 1985), which is believed to contain a $\sim 2.5 \times 10^{6} M_{\odot}$ black hole (Eckart \& Genzel 1996; Ghez et al. 1998). The coincidence in the geometry and kinematics of the southwestern edge of the molecular ring and ionized gas suggests that the ionized gas is dynamically coupled to the inner edge of the circumnuclear ring (e.g., Güsten et al. 1987).

On a larger scale, a nonthermal structure, Sgr A East, is thought to be a shell-type explosive event, possibly a supernova remnant (SNR), surrounded by the $50 \mathrm{~km} \mathrm{~s}^{-1}$ molecular cloud. A number of observations present strong evidence that these two objects are physically interacting with each other (e.g., Mezger et al. 1996; Zylka, Mezger, \& Wink 1990; Serabyn, Lacy, \& Achtermann 1992; Yusef-Zadeh et al. 1996). Thus, the dynamical coupling of the thermal Sgr A West and the CND, as well as the nonthermal Sgr A East and the $50 \mathrm{~km} \mathrm{~s}^{-1}$ cloud, has been fairly well established. A question that will be addressed in this paper is the nature of the interaction between these two systems.

Observations of molecular hydrogen gas are useful, not only for determining the dynamics of gas, but also for their potential for distinguishing shocked from UV-excited gas. Molecular hydrogen line emission has been detected from the inner edge of the circumnuclear ring. The excitation is thought to be produced by shocks driven into the ring by the ram pressure associated with the outflow from the IRS 16 cluster (Gatley et al. 1986; Yusef-Zadeh \& Wardle 1993). 
Broad emission lines observed toward the Galactic center could have contributions from outflows from the vicinity of the IRS 16 cluster with a terminal velocity $v_{w}=500-700 \mathrm{~km}$ $\mathrm{s}^{-1}$ and a mass-loss rate $\dot{M}_{w} \approx 4 \times 10^{-3} M_{\odot} \mathrm{yr}^{-1}$ (Hall, Kleinmann, \& Scoville 1982; Geballe et al. 1991; Allen, Hyland, \& Hiller 1990). However, the detection of shocked (as opposed to UV-heated) gas associated with the Galactic center molecular ring has been ambiguous. The intensity ratios of the $v=2-1$ and 1-0 S(1) lines of molecular hydrogen (Gatley et al. 1986; Burton \& Allen 1992, 1993; Ramsay-Howatt, Mountain, \& Geballe 1993; Pak, Jaffe, \& Keller 1996) are taken to be consistent with collisional excitation rather than fluorescence from low-density gas, but the high density of the molecular gas at the Galactic center and the intense UV radiation field in the region allow the line ratios from UV-irradiated gas to resemble those of shock-heated gas (Sternberg \& Dalgarno 1989; Burton, Hollenbach, \& Tielens 1990).

The $\mathrm{OH}$ molecule has recently proved to be useful in distinguishing shocked from radiatively excited molecular gas through the presence of $1720 \mathrm{MHz}$ masers. The presence of the $\mathrm{OH}(1720 \mathrm{MHz})$ maser line, and the absence of the $1665 / 1667 \mathrm{MHz}$ lines, provides a clear diagnostic of shocked molecular gas, since the far-IR radiation field from warm dust in UV-heated clouds would pump the latter transitions (Frail, Goss, \& Slysh 1994; Lockett, Gauthier, \& Elitzur 1999). Recent detection of diffuse X-ray emission from the interior of Sgr A East and from Sgr A West are consistent with the shock model enhancing the abundance of $\mathrm{OH}$ behind the shock front (e.g., Baganoff et al. 2001; Maeda et al. 2001; Wardle 1999). The expansion of a supernova remnant into the Sgr A East molecular cloud (i.e., the $50 \mathrm{~km} \mathrm{~s}^{-1}$ cloud) is believed to be responsible for the production of the $\mathrm{OH}(1720 \mathrm{MHz})$ maser emission (YusefZadeh et al. 1996, 1999b). If the OH $(1720 \mathrm{MHz})$ masers signify regions of shocked gas, this suggests that the $\mathrm{H}_{2}$ emission is also shock-excited.

In order to investigate this hypothesis, observations of the $\mathrm{H}_{2}$ 1-0 $S(1)$ and $\mathrm{Br} \gamma$ lines were carried out using the University of New South Wales Infrared Fabry-Perot (UNSWIRF) etalon on the Anglo-Australian Telescope (AAT) and NICMOS on the Hubble Space Telescope (HST) near the regions where $\mathrm{OH}(1720 \mathrm{MHz})$ masers are detected in the CND and Sgr A East. The NICMOS data provide subarcsecond spatial resolution, and the UNSWIRF data provide velocity information for both the molecular and ionized gas. Preliminary results of these observations combined with low-frequency radio continuum images were presented by Yusef-Zadeh et al. (1999c), who argued for the interaction of Sgr A East and the CND.

This paper presents the distribution of $\mathrm{H}_{2}$ gas in the CND in $\S 3.1$ and discusses the nature of $\mathrm{H}_{2}$ gas in $\S 3.2$, followed by the correlation of $\mathrm{H}_{2}$ linear feature, $\mathrm{OH}(1720$ $\mathrm{MHz}$ ) masers, the Sgr A East molecular cloud, and radio continuum emission from Sgr A East with each other, in $\S \S 3.3$ and 3.4. In particular, the evidence is presented that the $\mathrm{H}_{2}$ linear filament to the northwest of the CND is not only excited by Sgr A East but also associated with the CND by a ridge of molecular gas. We then present evidence that all Galactic center $\mathrm{OH}(1720 \mathrm{MHz})$ masers, with one exception, are accompanied by shocked $\mathrm{H}_{2}$ gas, with the implication that Sgr A East is responsible for shocking the gas in the CND and the 50 and $70 \mathrm{~km} \mathrm{~s}^{-1}$ molecular clouds. In $\S 3.5$, two extinction clouds are discussed toward the northern arm and Sgr A East before conclusions are drawn in $\S 4$.

\section{OBSERVATIONS}

Camera 3 of NICMOS on the HST was used to observe the 1-0 S(1) transition of $\mathrm{H}_{2}$ toward Sgr A West as well as Sgr A East. Twelve adjacent pointings, each with a 52" field of view and 0.203 pixel scale, were observed in $1 \%$ bandwidth filters containing the line + continuum $(\mathrm{F} 212 \mathrm{~N})$ and continuum (F215N). The observations were made on 1998 July 3 and 4, when the plate scale for camera 3 was 0 ".20384 $\times 0$.20310 along the detector axes in $x$ and $y$, respectively. Because the majority of the images had to be rotated by $\sim 135^{\circ}$ to orient north up, the $x$ and $y$ plate scales were effectively equally interpolated to an average value of 0.2035 pixel $^{-1}$ without the need to correct for nonsquare pixels. The individual pointings were additionally dithered four times in a square pattern spaced by $16^{\prime \prime}$ along the detector axes in order to provide spatial overlap, correct for bad pixels, and improve the sampling. Final mosaics covering $\sim 4^{\prime} \times 4^{\prime}$ were constructed by combining all 48 frames in each filter. Each of the 48 positions was observed for $128 \mathrm{~s}$ using a MULTI-ACCUM sequence to correct for cosmic rays and nonlinearity. The standard Space Telescope Science Institute (STScI) procedure CALNICA version 3.2 was used to reduce the data; this performs a bias subtraction, linearity correction, dark subtraction, and flat-field. A guide-star reaquisition during the six-orbit program forced the spacecraft orientation angle to change during the observation, and 8 of the 48 frames in each filter had to be rotated with respect to the rest. An IDL routine developed by the NICMOS team at the University of Arizona was used to make the mosaic in each filter, and overlapping good pixels were median combined. Bicubic interpolation was used to shift and rotate the images. The effective plate scale is 0.2035 .

The NICMOS $\mathrm{H}_{2}$ image is constructed, in principle, by subtracting an appropriately flux-calibrated $\mathrm{F} 215 \mathrm{~N}$ mosaic from the F $212 \mathrm{~N}$ mosaic. However, a simple subtraction did not yield a satisfactory line image, and additional steps were taken, as described here. The thermal background (as measured in regions devoid of stars due to high extinction) was removed from the data; DC levels of $0.61 \mathrm{ADU} \mathrm{s}^{-1}$ and $0.71 \mathrm{ADU} \mathrm{s}^{-1}$ were subtracted for $\mathrm{F} 212 \mathrm{~N}$ and $\mathrm{F} 215 \mathrm{~N}$, respectively. Random fluctuations in DC levels by quadrant in the NICMOS detectors contributed to another (much fainter) DC background that needed to be subtracted in rectangular patches; this subtle effect was only apparent in the F212N-F215N difference image and could not be perfectly corrected because of the contamination by the stars that dominate both mosaics. Next, the F215N images were scaled to the F212N image in order to minimize the stellar residuals upon subtraction; the scale factor used was 1.02. Both positive and negative stellar residuals remained, however, because of intrinsic variations in stellar colors (mostly due to patchy extinction) and because of pointspread function (PSF) differences in alignment and illumination of the undersampled pixels. In order to remove the high spatial frequency residuals and to improve the signalto-noise ratio $(\mathrm{S} / \mathrm{N})$, a $5 \times 5$ pixel median filter was applied to the difference image, excluding very negative pixels from the median procedure. The resulting spatial resolution obtained after medianing was estimated by measuring the effect of the same median on the continuum image: stars 
were measured to increase in FWHM from 0 ".5 to 0 ".7. The reduction in flux of individual bright pixels in the $\mathrm{H}_{2}$ image due to the median filter was measured to be not more than a factor of 2.5. The flux in $1^{\prime \prime}$ apertures remained constant after applying the median filter. Finally, the difference image was converted to line flux units with the calibration supplied by STScI. Because of the leakage of blueshifted $\mathrm{Br}$ gamma emission into the $2.15 \mu$ m continuum filter, portions of the "minispiral" of ionized gas within the inner parsec show up as negative in the $\mathrm{H}_{2}$ image (masked to zero is equivalent to white in the $\mathrm{H}_{2}$ image). The final NICMOS $\mathrm{H}_{2}$ mosaic is shown below in reverse gray scale in Figures $2 a, 2 b, 3$, and 7 , and the features discussed in this paper are labeled in Figure $2 b$. Registration of the NICMOS data with the radio data was achieved by aligning position of IRS 7, the brightest continuum source in the NICMOS image, with its radio maser position. The position of Sgr A* is taken to be that given in Menten et al. (1997). Alignment accuracy is estimated to be correct to better than 1 NICMOS pixel (0".2).

Observations of the $2.122 \mu \mathrm{m}$ line of $\mathrm{H}_{2}$ were also obtained with the AAT in 1998 June, using the IRIS 1-2.5 $\mu \mathrm{m}$ camera in conjunction with the UNSWIRF etalon (Ryder et al. 1998). With a FWHM spectral resolution of $\sim 75 \mathrm{~km} \mathrm{~s}^{-1}$, a pixel size of 0 "'.77, and a $100^{\prime \prime}$ circular field of view, the etalon is scanned through a spectral line of interest with a $40 \mathrm{~km} \mathrm{~s}^{-1}$ plate spacing, and with an "off-line" setting chosen to provide continuum subtraction. Sky frames are also taken for each etalon spacing.

Four positions were observed, F1 to F4, with central positions at $17^{\mathrm{h}} 42^{\mathrm{m}},-28^{\circ}(1950)$ of $\left(30^{\mathrm{s}}, 58^{\prime} 45^{\prime \prime}\right),\left(28^{\mathrm{s}}\right.$, $\left.59^{\prime} 40^{\prime \prime}\right),\left(33^{\mathrm{s}},-29^{\circ} 00^{\prime} 10^{\prime \prime}\right)$, and $\left(34.5,59^{\prime} 50^{\prime \prime}\right)$, respectively. These are shown in Figure 1, overlaid on a $K$-band (2.15 $\mu \mathrm{m})$ continuum image of the Galactic center from NICMOS. A mosaic of the four frames was carried out using FLATN in AIPS, and the gray-scale image of integrated line emission is displayed in Figure $2 c$. The 6, 11, 6, and 7 plate spacings were taken at each position, covering $200,395,200$, and $240 \mathrm{~km} \mathrm{~s}^{-1}$, respectively. Integration time per frame was 2 minutes. Seeing varied between $2^{\prime \prime}$ and $3^{\prime \prime}$ during the observations.

Data reduction was through a custom software page using IRAF. ${ }^{1}$ Frames are linearized, flat-fielded using a dome flat, sky-subtracted, shifted to align the stars in each frame, smoothed, and the off-line frame subtracted from each on-line frame (each having been appropriately scaled to minimize residuals from the subtraction process). Stacking the frames yields a data cube, which is then fitted pixelby-pixel with the instrumental profile (a Lorentzian) to yield a line image. Furthermore, the line center is also determined from the peak position of the fitted line. The data cube obtained is not a true velocity-position cube, since the wavelength associated with each pixel (as well as the spectral resolution) varies across the array (by up to $40 \mathrm{~km} \mathrm{~s}^{-1}$ ), but the variation can be calibrated by observation of an arc line, and hence the line center at each pixel determined. Typically this is accurate to $\sim 10 \mathrm{~km} \mathrm{~s}^{-1}$, but in the crowded environment of the Galactic center, continuum subtraction was imperfect and accuracy varies across the field. Absolute wavelength calibration is then made by comparison to the etalon setting for the $\mathrm{H}_{2}$ emission in known

\footnotetext{
${ }^{1}$ Image Reduction and Analysis Facility; see http://www.iraf.noao.edu.
}

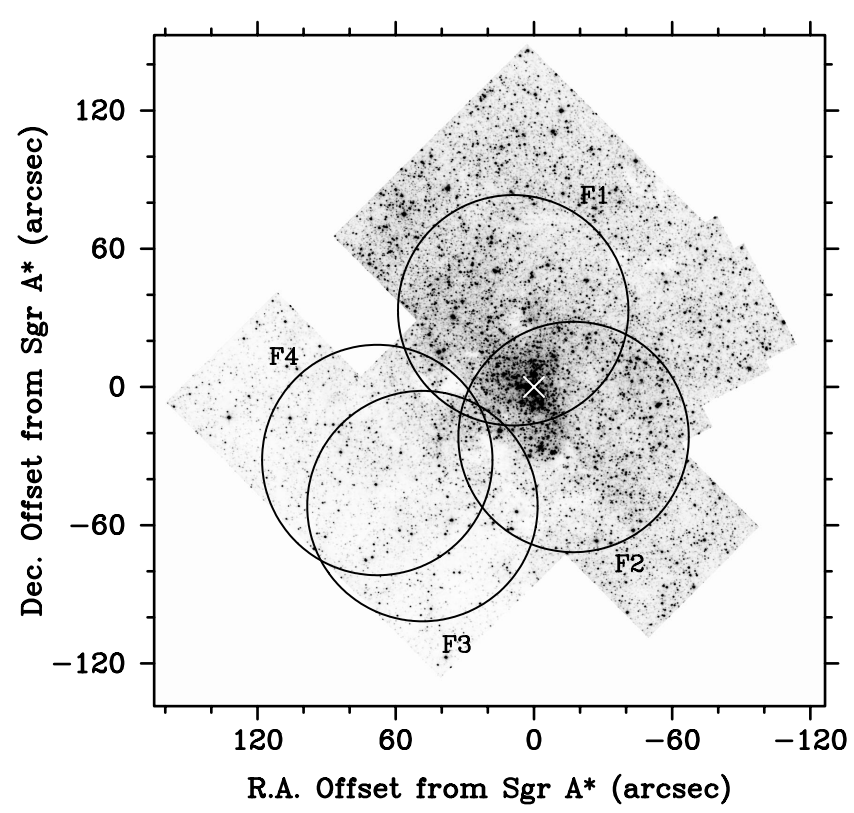

FIG. 1. $-2.15 \mu \mathrm{m}$ continuum image of the Galactic center (from NICMOS observations) overlaid with the four fields (F1 to F4) observed in $\mathrm{H}_{2}$ using the AAT/UNSWIRF. The circles mark the center of the $100^{\prime \prime}$ diameter frames. The Galactic plane is apparent running northeastsouthwest across the image. Offsets in arcseconds for circular field centers from Sgr A* are: F1 (9.0, 33.3), F2 (-17.2, -21.7), F3 (48.4, - 51.7), and F4 $(68.3,-31.7)$. The gray scale in the image ranges from -5 to $500 \mu \mathrm{Jy}$ pixel $^{-1}$. The noise level is $1.5 \mu \mathrm{Jy}$ pixel $^{-1}$, and the peak pixel at IRS 7 (which is not saturated) is at level of $0.12 \mathrm{Jy} \mathrm{pixel}^{-1}$. The flux of IRS 7 at $2.15 \mu \mathrm{m}$ is measured to be $0.95 \mathrm{Jy}$, or $7.2 \mathrm{mag}$. Stars as faint as 15 th magnitude are measured in uncrowded regions of high extinction. The white cross coincides with the position of Sgr A*.

sources, in this case IC 4406 (at $\left.-41 \mathrm{~km} \mathrm{~s}^{-1} V_{\mathrm{LSR}}\right)$ and M17 $\left(+21 \mathrm{~km} \mathrm{~s}^{-1} V_{\mathrm{LSR}}\right)$. The overall accuracy in the line center determination is estimated to be $\sim 25 \mathrm{~km} \mathrm{~s}^{-1}$. The moderate spectral resolution, and the wide wings of the instrumental profile, preclude any attempt to determine further spectral information other than the line center. Flux calibration was made by comparison to the star HR 6378 with a $K$-band magnitude of $2.29 \mathrm{mag}$, and the absolute accuracy is typically around $30 \%$ in the line fluxes. An image was also obtained in the $2.166 \mu \mathrm{m} \mathrm{Br} \gamma$ hydrogen recombination line in 1999 June, with a widely spaced velocity channel separation of $95 \mathrm{~km} \mathrm{~s}^{-1}$. While a detailed discussion of these results will be given elsewhere, selected images from these data are used in this paper.

\section{RESULTS AND DISCUSSION}

\section{1. $\mathrm{H}_{2}$ Gas in the Circumnuclear Ring}

The region observed with UNSWIRF is shown in Figure 1 , with the four positions marked by circles overlaid on a 2.2 $\mu \mathrm{m}$ NICMOS continuum image of the Galactic center. Figure $2 a$ shows the $\mathrm{H}_{2}$ image obtained with NICMOS, overlaid with the outermost contour from the $88.6 \mathrm{GHz}$ $J=1-0$ line map of HCN (Güsten et al. 1987), which delineates the extent of dense gas in the CND. The $\mathrm{H}_{2}$ emission is shown in reverse gray scale. Also marked are the positions of $\mathrm{OH}(1720 \mathrm{MHz})$ masers (from Yusef-Zadeh et al. 1996) and Sgr A*. Although the median filtering that was applied twice removed many of the stellar residuals due to the undersampled pixels, some artifacts remain. Nevertheless, the overall spatial distribution of the $\mathrm{H}_{2}$ emission is 


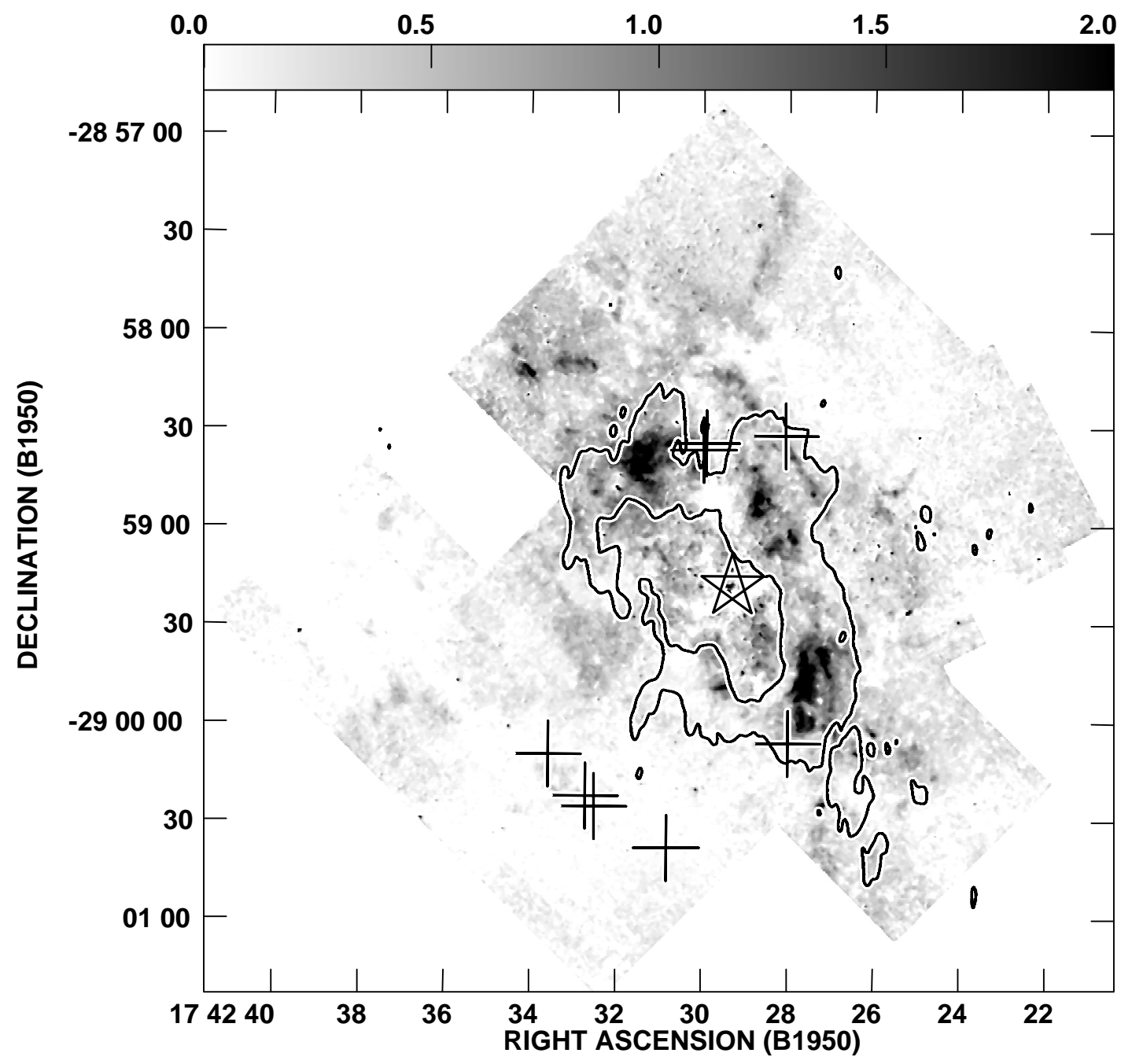

FIG. $2 a$

FIG. 2.-(a) Continuum-subtracted $\mathrm{H}_{2}$ 1-0 S(1) line $2.12 \mu \mathrm{m}$ image of the Galactic center obtained with HST/ NICMOS. Contours indicate the extent of the HCN $J=1-0$ line $88.6 \mathrm{GHz}$ emission from the inner region of the circumnuclear ring, as mapped by Güsten et al. (1987). The star coincides with the position of Sgr A*. Despite the high spatial resolution of the HST image, and the stability of the point-spread function, this image illustrates the extreme difficulty in obtaining a line image in a crowded region when only low $(1 \%)$ spectral resolution is used. The $\mathrm{H}_{2}$ emission is shown in reverse gray scale. $(b)$ Prominent features are labeled on this NICMOS image of $\mathrm{H}_{2}$ line emission. The reverse gray scale ranges from -0.05 to $1.5 \times 10^{-16} \mathrm{erg} \mathrm{cm}^{-2} \mathrm{~s}^{-1} \mathrm{pixel}^{-1}$ $\left(1 \mathrm{pixel}=0^{\prime \prime}\right.$.2035), and is chosen to highlight the fainter features. The noise level is of the order of $0.02 \times 10^{-16} \mathrm{erg} \mathrm{cm}^{-2} \mathrm{~s}^{-1}$ pixel ${ }^{-1}$, and the brightest clumps peak at 3.8 in these units, yielding a peak $\mathrm{S} / \mathrm{N}$ of 190 . The plus signs show the positions of the 134 and $43 \mathrm{~km} \mathrm{~s}{ }^{-1} \mathrm{OH}(1720 \mathrm{MHz}) \mathrm{maser} \mathrm{B}$ and C, respectively, from Yusef-Zadeh et al. (1996), as well as the $-132 \mathrm{~km} \mathrm{~s}^{-1}$ maser feature in the south lobe (Yusef-Zadeh et al. 2001). (c) Mosaic image of four UNSWIRF frames (F1 to F4) showing gray scale and contours of integrated $\mathrm{H}_{2} S(1) 1-0$ line emission. The square in the ring is due to artifacts of mosaicing. (In subsequent figures plus signs mark the position of $\mathrm{OH}(1720 \mathrm{MHz})$ masers.)

clear, and many individual features are clearly resolved. Figures $2 b$ and $2 c$ present the prominent $\mathrm{H}_{2}$ features labeled on an NICMOS $\mathrm{H}_{2}$ image and the mosaic of four UNSWIRF images of $\mathrm{H}_{2}$ line intensity, respectively. The strongest $\mathrm{H}_{2}$ emission is associated with the northeast and southwest lobes of the CND, as first mapped by Gatley et al. $(1984,1986)$. The peak $\mathrm{H}_{2}$ intensities in the northeast and southwest are 7 and $8 \times 10^{-15} \mathrm{erg} \mathrm{s}^{-1} \mathrm{~cm}^{-2} \operatorname{arcsec}^{-2}$, respectively. Apart from the CND, the most striking feature is a linear filament to the northwest of the CND. Centered on $\alpha=17^{\mathrm{h}} 42^{\mathrm{m}} 28^{\mathrm{s}} 5, \delta=-28^{\circ} 58^{\prime} 30^{\prime \prime}$, and extending about $1^{\prime}$ in a northeast-southwest direction, the FWHM of the width of the filament is only $\sim 1$.'3 $(0.05 \mathrm{pc}$ at the distance of $8 \mathrm{kpc}$ ). Several compact knots of emission can be found along the length of the filament. The most pointlike of these is found at the filament's southwest end and is located 24".8 west, 28 ". 3 north of Sgr A*. Since there is no detected stellar counterpart in the continuum filter to this compact $\mathrm{H}_{2}$ knot, it is clearly not an artifact of improper stellar subtraction; therefore, it is likely nonstellar in origin despite its pointlike appearance. The typical intensity of the $\mathrm{H}_{2}$ emission along the linear feature is between $\sim 2$ and $3 \times 10^{-15}$ $\mathrm{erg} \mathrm{s}^{-1} \mathrm{~cm}^{-2} \operatorname{arcsec}^{-2}$. There is a gap in the filament near $\alpha=17^{\mathrm{h}} 42^{\mathrm{m}} 29^{\mathrm{s}}, \delta=-28^{\circ} 58^{\prime} 30^{\prime \prime}$.

Contours of radio continuum emission at $6 \mathrm{~cm}$ obtained with the Very Large Array of the National Radio Astronomy Observatory ${ }^{2}$ (Yusef-Zadeh \& Morris 1987; Yusef-Zadeh \& Wardle 1993) are superimposed on the NICMOS $\mathrm{H}_{2}$ emission in Figure 3. The distribution of ionized gas near the north and south lobes is asymmetric,

${ }^{2}$ The National Radio Astronomy Observatory is a facility of the National Science Foundation, operated under a cooperative agreement by Associated Universities, Inc. 


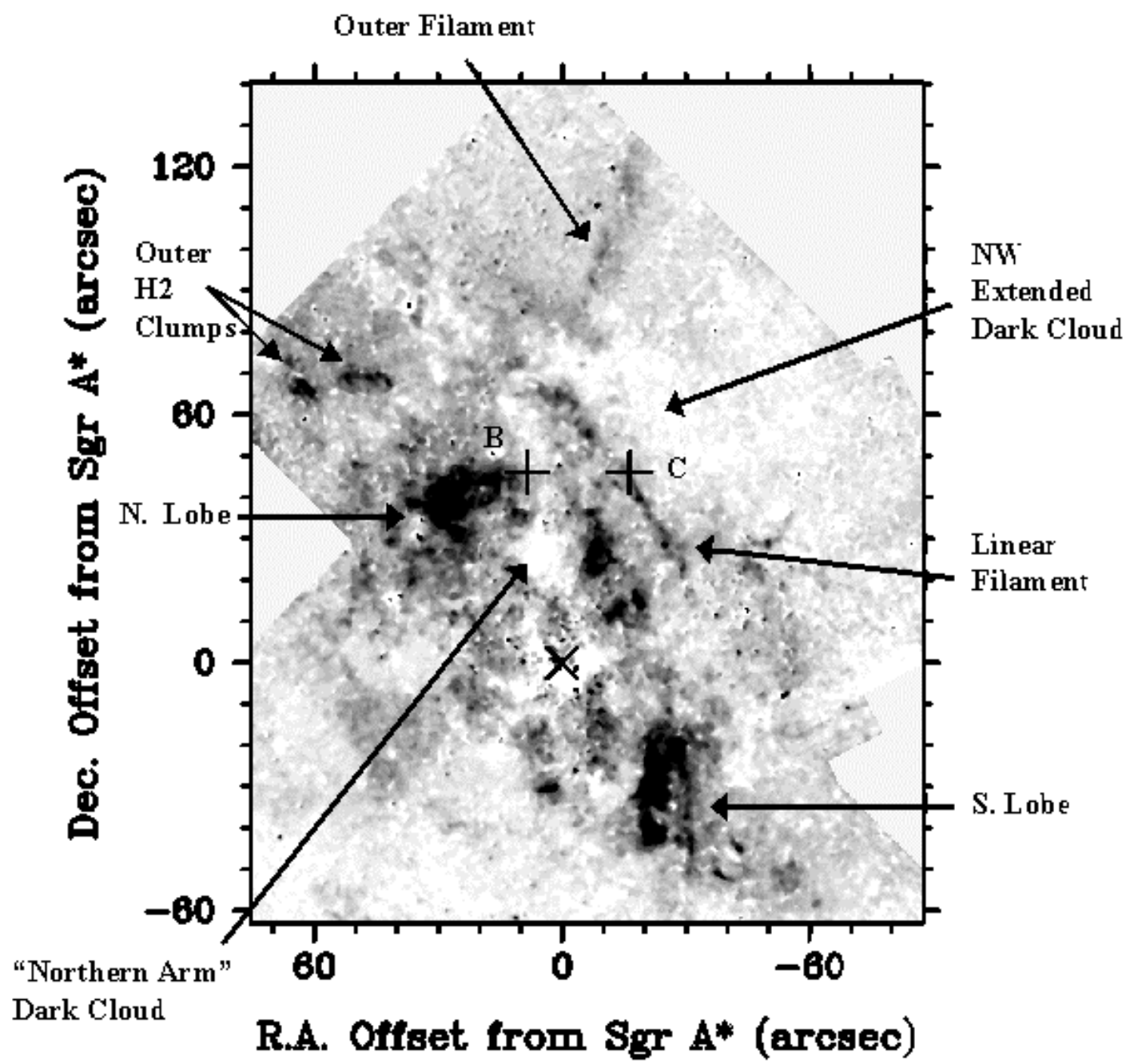

FIG. $2 b$

and the spiral-shaped structure of Sgr A West is evident near the center of the image. Figure 4 shows the distribution of molecular hydrogen emission overlaid with contours of $\mathrm{Br} \gamma$ emission extracted from the data cube at 125 and -160 $\mathrm{km} \mathrm{s}^{-1}$. As expected, the distribution of $\mathrm{Br} \gamma$ line emission broadly follows the radio free-free emission, the lowest contour following the edge of the south lobe of the CND in Figure $4 b$ but avoiding the north lobe in Figure $4 a$. The intensity of $\mathrm{Br} \gamma$ emission is stronger in the north than in the south lobe by a factor of about 3 , peaking at $2 \times 10^{-14} \mathrm{erg}$ $\mathrm{s}^{-1} \mathrm{~cm}^{-2} \operatorname{arcsec}^{-2}$ in the north and $6 \times 10^{-15} \mathrm{erg} \mathrm{s}^{-1}$ $\mathrm{cm}^{-2} \operatorname{arcsec}^{-2}$ in the south. The brightest $\mathrm{Br} \gamma$ emission arises from the minicavity with the flux of $3 \times 10^{-14} \mathrm{erg} \mathrm{s}^{-1}$ $\mathrm{cm}^{-2} \operatorname{arcsec}^{-2}$ (Roberts, Yusef-Zadeh, \& Goss 1996) thatlies near Sgr A*. The emission velocities for the $\mathrm{Br} \gamma$ features peak at $-350,+29$, and -160 for the minicavity, north arm, and south arm of Sgr A West, respectively (a more detailed discussion of $\mathrm{Br} \gamma$ data will be given elsewhere).

Figure $5 a$ shows the distribution of the integrated intensity of the $\mathrm{H}_{2}$ 1-0 S(1) line for field 1 (F1), which includes the northern part of the CND and the newly discovered filament to its northwest. Figure $5 b$ shows the same image superimposed on a $2.2 \mu \mathrm{m}$ continuum image, and also has the line center velocities for the principle emission features labeled, in addition to the $\mathrm{OH} 1720 \mathrm{MHz}$ masers. The brightest clump in the north lobe, with $V_{\mathrm{LSR}}$ velocity of $110-115 \mathrm{~km} \mathrm{~s}^{-1}$, has a peak intensity of $6.6 \times 10^{-15} \mathrm{erg}$ $\mathrm{cm}^{-2} \mathrm{~s}^{-1} \operatorname{arcsec}^{-2}$. The $134 \mathrm{~km} \mathrm{~s}{ }^{-1} \mathrm{OH}$ maser (B) lies adjacent to the western edge of the north lobe.

The $\mathrm{H}_{2}$ linear filament is brightest at $\approx 75 \mathrm{~km} \mathrm{~s}^{-1}$, but the line center velocity decreases to $\sim 50 \mathrm{~km} \mathrm{~s}^{-1}$ along its northeast extension. The western edge of the CND (sometimes called the western arc) appears at velocities ranging between 55 and $80 \mathrm{~km} \mathrm{~s}^{-1}$. The $\mathrm{OH}(1720 \mathrm{MHz})$ masers in this vicinity are distributed along the edges of the north lobe and the filament where the intensity of $\mathrm{H}_{2}$ emission falls from the peak values, to levels of $1-2 \times 10^{-15} \mathrm{erg}$ $\mathrm{cm}^{-2} \mathrm{~s}^{-1} \operatorname{arcsec}^{-2}$. At the position of the $43 \mathrm{~km} \mathrm{~s}^{-1}$ maser (C) a ridge of diffuse $\mathrm{H}_{2}$ emission appears to connect the filament to the western edge of the CND. However, the interpretation at this location is confused by the presence of the " $70 \mathrm{~km} \mathrm{~s}^{-1}$ cloud" seen in [O I] $63 \mu \mathrm{m}$ (Jackson et al. 1993), a feature known to be associated with the CND. The kinematics of the $\mathrm{H}_{2}$ gas in the filament and the ridge, with velocities around $60 \mathrm{~km} \mathrm{~s}^{-1}$, suggests that the $\mathrm{H}_{2}$ emission from these features is indeed associated with the $70 \mathrm{~km} \mathrm{~s}^{-1}$ cloud.

A plumelike feature within the CND, as shown in Figure $5 a$, also extends from just northeast of Sgr A* near IRS 7, located at $\alpha=17^{\mathrm{h}} 42^{\mathrm{m}} 30^{\mathrm{s}} \cdot 2, \delta=-28^{\circ} 59^{\prime} 3^{\prime \prime}$. This new feature peaks at emission velocities near $80 \mathrm{~km} \mathrm{~s}^{-1}$, with a peak 


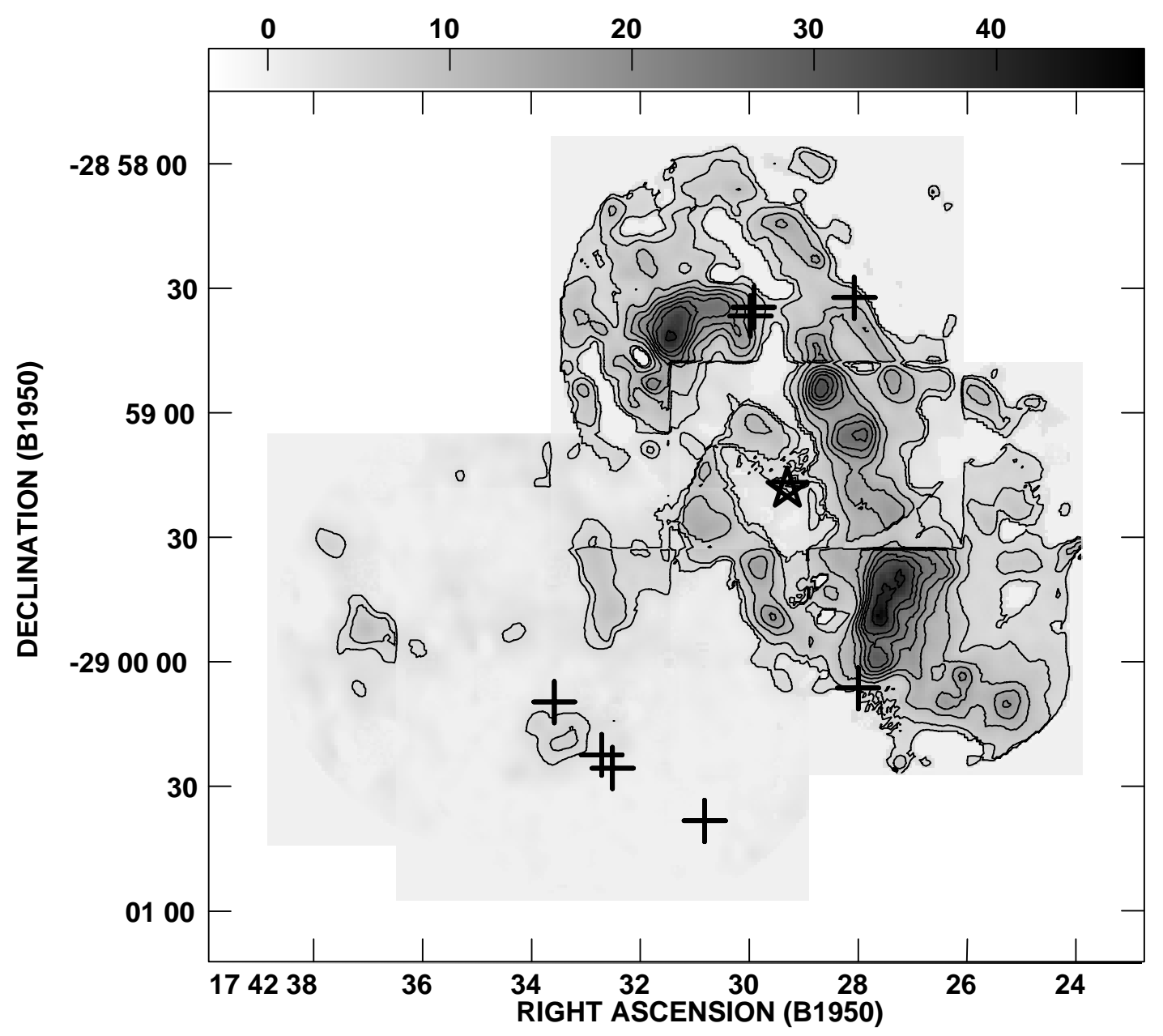

FIG. $2 c$

flux of $1.5 \times 10^{-15} \mathrm{erg} \mathrm{cm}^{-2} \mathrm{~s}^{-1} \operatorname{arcsec}^{-2}$. It does not appear to be associated with any ionized streamers of Sgr A West, but is adjacent to the [O I] $63 \mu \mathrm{m}$ peak detected by Jackson et al. (1993) at similar velocities.

$\mathrm{H}_{2}$ emission is also present on the negative Galactic longitude side of the CND. The maps in Figures $6 a$ and $6 b$ show the line intensity in field 2 (F2), centered near the southern lobe of the CND. The brightest clump of the south lobe, with $V_{\mathrm{LSR}}$ velocity of $-40 \mathrm{~km} \mathrm{~s}^{-1}$, has a peak intensity of $7.6 \times 10^{-15} \mathrm{erg} \mathrm{cm}^{-2} \mathrm{~s}^{-1} \operatorname{arcsec}^{-2}$. Weak, diffuse $\mathrm{H}_{2}$ emission is also present outside and inside the CND, with intensities $\sim 1 \times 10^{-15} \mathrm{erg} \mathrm{cm}^{-2} \mathrm{~s}^{-1} \operatorname{arcsec}^{-2}$. Within the CND, as outlined by the HCN emission (Wright et al. 1987), there is diffuse $\mathrm{H}_{2}$ emission, at positive velocities. Diffuse emission also extends beyond the CND, to the southwest, with both negative and positive velocity components. It is clear that there is $\mathrm{H}_{2}$ gas within the molecular cavity and that its kinematics on the negative-longitude side of the CND is inconsistent with the sense of rotation of the circumnuclear ring.

\subsection{The Nature of $\mathrm{H}_{2}$ Emission from the Circumnuclear Ring}

Our UNSWIRF and NICMOS observations detect peak fluxes in the 1-0 $S(1)$ molecular hydrogen line of approximately $7 \times 10^{-15} \mathrm{erg} \mathrm{s}^{-1} \mathrm{~cm}^{-2} \operatorname{arcsec}^{-2}$ in the north and south lobes. This is comparable to the fluxes reported by
Gatley et al. (1986; $\approx 4 \times 10^{-15} \mathrm{erg} \mathrm{s}^{-1} \mathrm{~cm}^{-2} \operatorname{arcsec}^{-2}$ in an $18^{\prime \prime}$ diameter aperture) but somewhat more than those of Burton \& Allen $\left(1992 ; \approx 2 \times 10^{-15} \mathrm{erg} \mathrm{s}^{-1} \mathrm{~cm}^{-2} \operatorname{arcsec}^{-2}\right.$ in a 1".4 by 4.7 east-west aperture placed, but not peaked, on the north lobe). Adopting $A_{K}=3$, the extinctioncorrected line intensity from the lobes is approximately $4 \times 10^{-3} \mathrm{erg} \mathrm{s}^{-1} \mathrm{~cm}^{-2} \mathrm{sr}^{-1}$ at its peak. The extinction, of course, could be higher toward the north and south lobes, as recently discussed by Stolovy, Scoville, \& Yusef-Zadeh (2001).

The distribution of emission is broadly consistent with the orientation of the inner circumnuclear disk inferred from HCN observations (Güsten et al. 1987; Jackson et al. 1993): a $0.5 \mathrm{pc}$ thick torus of emission with normal surface intensity $\sim 10^{-3} \mathrm{erg} \mathrm{cm}^{-2} \mathrm{~s}^{-1} \mathrm{sr}^{-1}$ inclined at $70^{\circ}$ to the line of sight will produce the north and south lobes by limb brightening. The source of excitation of the $\mathrm{H}_{2}$ emission is puzzling. Gatley et al. (1984) argued that the inner edge of the circumnuclear ring is shocked by a wind from the masslosing stars in the central few parsecs, for which $\dot{M} \sim 3$ $\times 10^{-3} M_{\odot} \mathrm{yr}^{-1}$ and $v_{\text {wind }} \approx 750 \mathrm{~km} \mathrm{~s}^{-1}$ (Krabbe et al. 1991; Najarro et al. 1997). At 1.7 pc, this combined wind would be capable of driving a shock of speed $v_{s}$ into a preshock medium of $\mathrm{H}$ density $n_{\mathrm{H}}$ with $n_{\mathrm{H}} v_{s}^{2} \approx 2 \times$ $10^{6} \mathrm{~cm}^{-3} \mathrm{~km}^{2} \mathrm{~s}^{-2}$. However, the observed intensity can be produced by C-type shock waves with $v_{s} \approx 30 \mathrm{~km} \mathrm{~s}^{-1}$ if the preshock density is $\sim 10^{4} \mathrm{~cm}^{-3}$, or in both C- and J-type shocks with $v_{s} \approx 20 \mathrm{~km} \mathrm{~s}^{-1}$ if the preshock density is $\gtrsim 10^{5}$ 


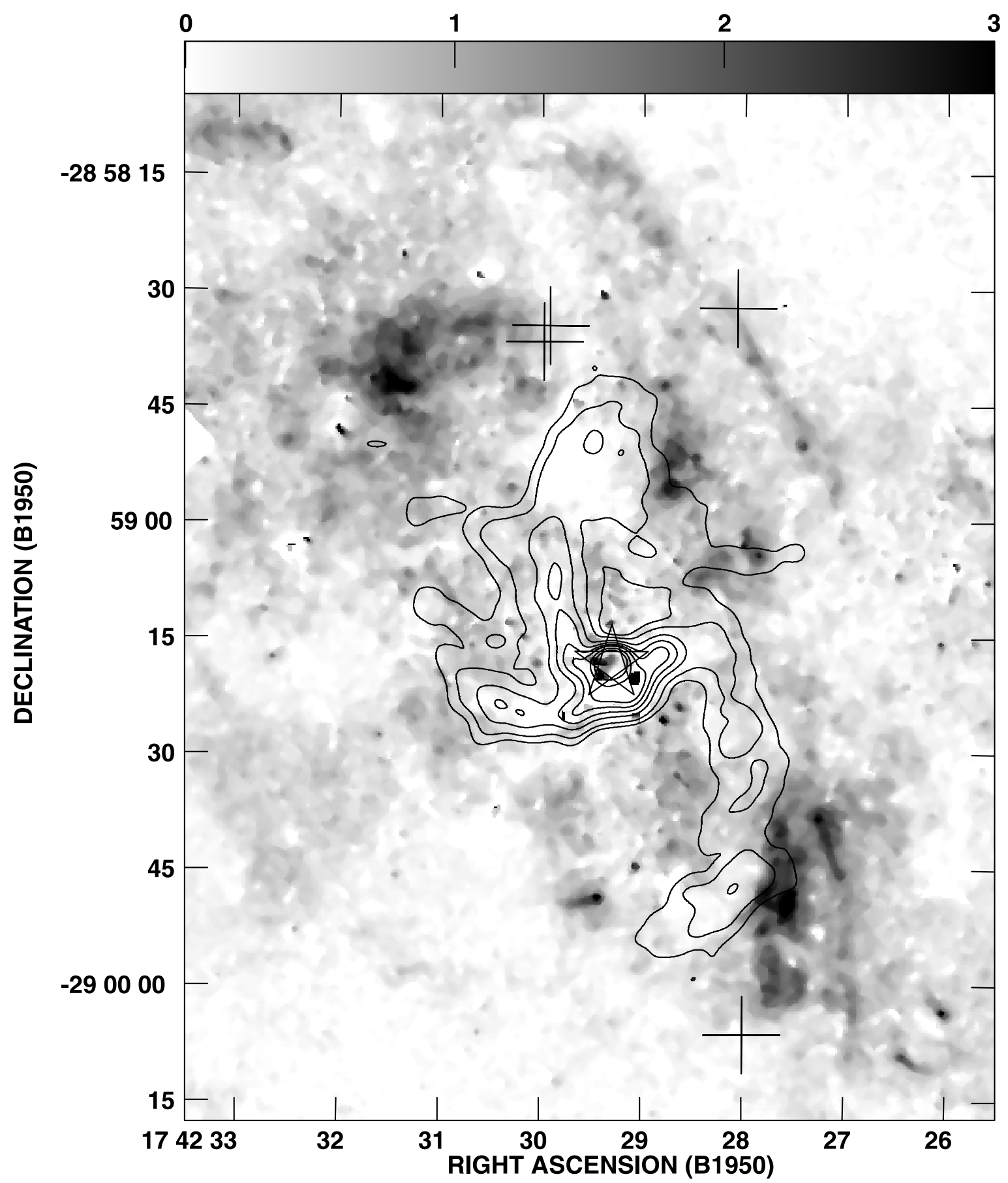

Fig. 3. - Contours of $6 \mathrm{~cm}$ radio continuum emission from Sgr A West, obtained with a resolution of 3".4 $\times 2$ ".9 and set at (75, 100, 150, 200, 250, 300, 400, $500) \times 1 \mathrm{mJy}$, superimposed on a gray-scale NICMOS image of the $\mathrm{H}_{2} 1-0 S(1)$ line emission from the CND. The star shows the position of Sgr A*.

$\mathrm{cm}^{-3}$ (Kwan 1977; Draine, Roberge, \& Dalgarno 1983; Kaufman \& Neufeld 1996a). Thus, the ram pressure of the IRS 16 wind is at most one-fifth of that required. ${ }^{3}$

The intense UV field $\left(G_{0} \sim 10^{5}\right)$ at the Galactic center (Sternberg \& Dalgarno 1989; Burton et al. 1990) is another source of excitation, although UV may be prevented from reaching the eastern side of the ring by intervening material

\footnotetext{
${ }^{3}$ Gatley et al. (1984) conclude that this ram pressure is sufficient, but this is based on an estimate that the $\sim 50 L_{\odot}$ emitted in the 1-0 S(1) line arises from $\sim 0.01 M_{\odot}$ of gas at $2000 \mathrm{~K}$, which appears to be a factor of 10 too low.
}

associated with the northern arm (Genzel, Hollenbach, \& Townes 1995). In equilibrium, a photodissociation region (PDR) emits $\sim 10^{-3} \mathrm{erg} \mathrm{cm}^{-2} \mathrm{~s}^{-1} \mathrm{sr}^{-1}$ in the 1-0 S(1) line only if the gas density $\gtrsim 10^{7} \mathrm{~cm}^{-3}$, but then the predicted $[\mathrm{O} \mathrm{I}] 63 \mu \mathrm{m}$ intensity $\left(\gtrsim 0.1 \mathrm{erg} \mathrm{cm}{ }^{-2} \mathrm{~s}^{-1} \mathrm{sr}^{-1}\right.$; Burton et al. 1990) is several times higher than observed (Jackson et al. 1993). Although the presence of high-density gas $\left(n \sim 10^{6}\right.$ $10^{8} \mathrm{~cm}^{-3}$ ) at the inner edge of the circumnuclear ring has been inferred from HCN observations (Güsten et al. 1987; Jackson et al. 1993; Marshall, Lasenby, \& Harris 1995), this material has a sky-covering fraction $\sim 0.1$, and so the $\mathrm{H}_{2}$ line intensity would be diluted by this factor. In fact, the 


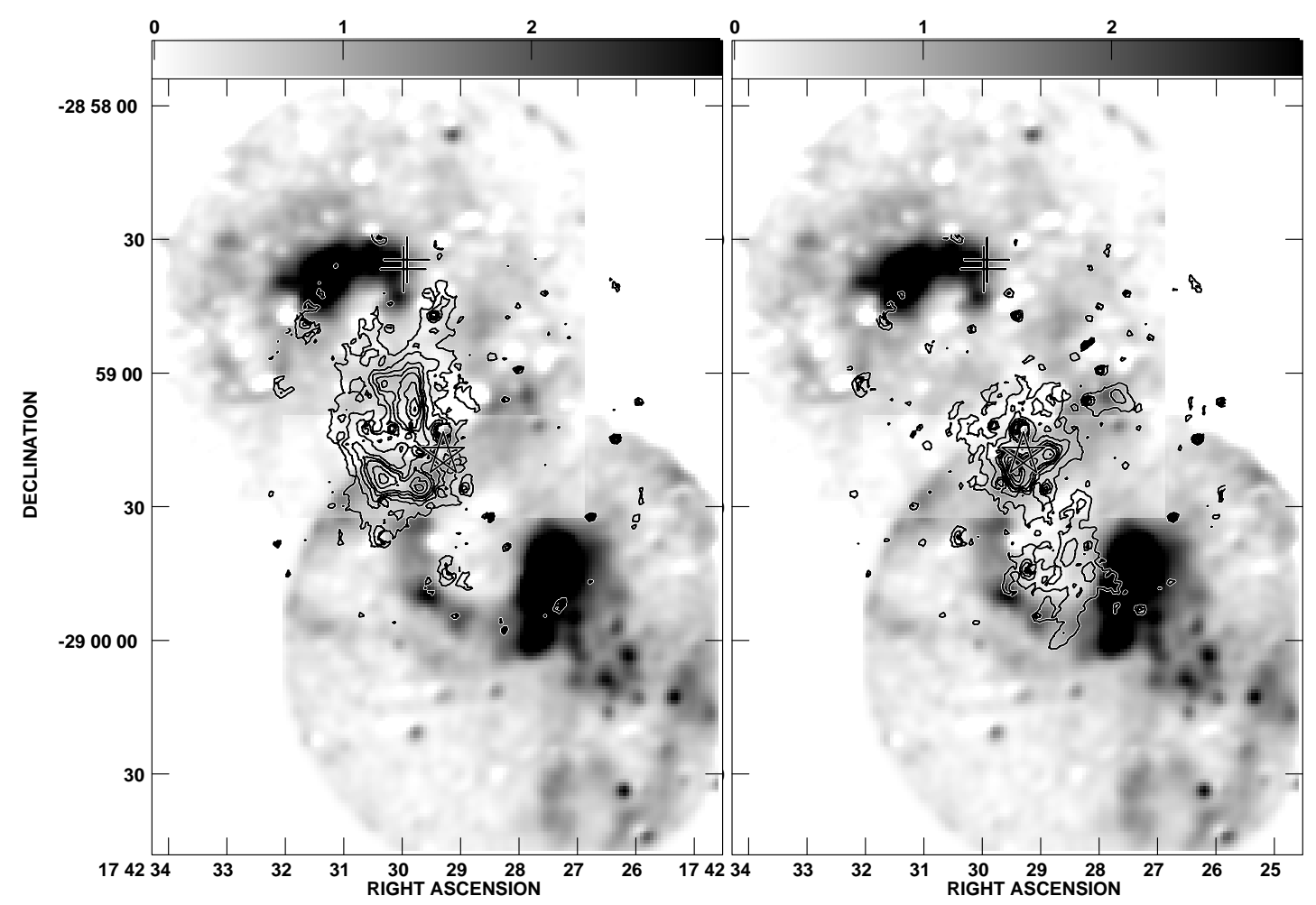

Fig. 4.-Gray scale: Left and right panels show $\mathrm{H}_{2}$ 1-0 S(1) emission from the north and south lobes, respectively, in reverse gray scale, extracted from two frames of the UNSWIRF data cubes (F1 at $125 \mathrm{~km} \mathrm{~s}^{-1}$ and F2 at $-85 \mathrm{~km} \mathrm{~s}^{-1}$ for the north and south lobes of the circumnuclear ring, respectively). The location of frames F1 and F2 can be seen in Fig. 1. Contours: Br $\gamma$ emission extracted from the data cube at $125 \mathrm{~km} \mathrm{~s}^{-1}$ is superimposed in the left panel, -160 $\mathrm{km} \mathrm{s}^{-1} \mathrm{Br} \gamma$ emission is superimposed in the right panel. As discussed in the text, these are not true velocity channel frames, but do give an indication of the morphology of the line emission at these velocities. Contour levels are $\left(1,2,3,4,5,7,9,22,25,20,30\right.$, and 40) times $5 \times 10^{-16} \mathrm{erg} \mathrm{s}^{-1} \mathrm{~cm}^{-2}$ arcsec ${ }^{-2}$. Plus signs mark the location of compact OH (1720) masers (source B from Yusef-Zadeh et al. 1996) associated with the north lobe, emitting at $134 \mathrm{~km} \mathrm{~s}^{-1}, V_{\mathrm{LSR}}$. The star shows the position of Sgr A*.

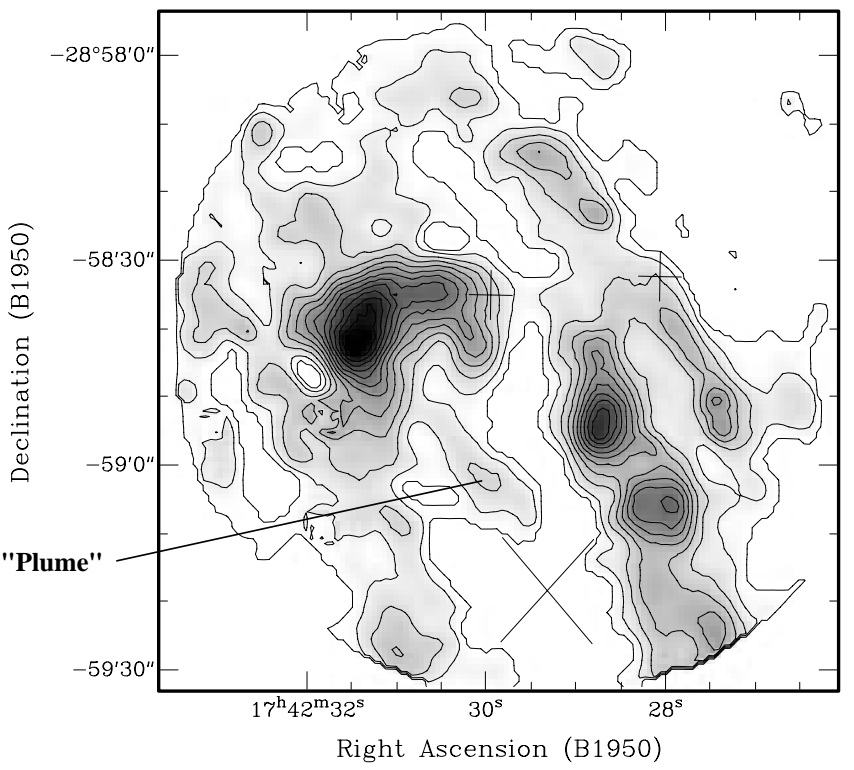

FIG. $5 a$

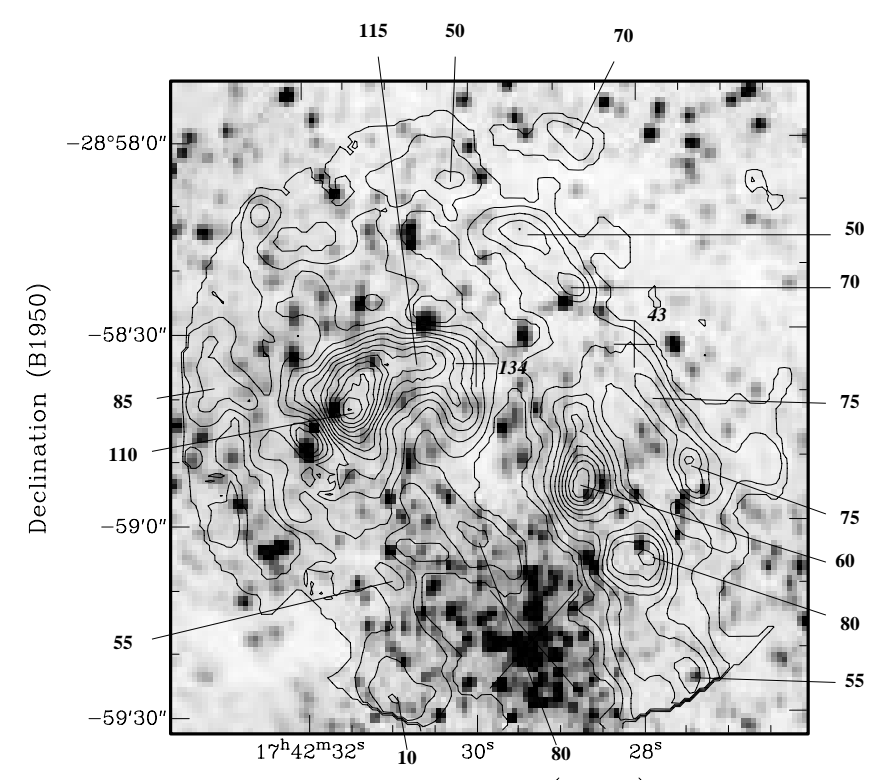

Right Ascension (B1950)

FIG. $5 b$

FIG. 5.- (a) Contours of the integrated $2.12 \mu \mathrm{m} \mathrm{H}_{2} 1-0 S(1)$ line emission from UNSWIRF field 1 of Fig. 1, showing the northern portion of the CND. Contours are overlaid on a gray-scale representation of the same data. Contour levels start at, and are in increments of, $5 \times 10^{-16} \mathrm{erg} \mathrm{s}^{-1} \mathrm{~cm}^{-2}$ arcsec ${ }^{-2} .(b)$ Same as (a), except that the contours are overlaid on a $2.2 \mu \mathrm{m}$ continuum image of the region. Labeled, around the edges of the image, are the line center velocities in $\mathrm{km} \mathrm{s}^{-1}\left(V_{\mathrm{LSR}}\right)$ of prominent features. Plus signs indicate the locations of $\mathrm{OH} 1720 \mathrm{MHz}$ masers, labeled with their associated emission velocity, and the cross shows the location of Sgr A*. The brightest $2 \mu \mathrm{m}$ source, IRS 7, lies $5^{\prime \prime}$ to the north of Sgr A*. The central velocities of emission features are labeled to the nearest $5 \mathrm{~km} \mathrm{~s}^{-1}$. 


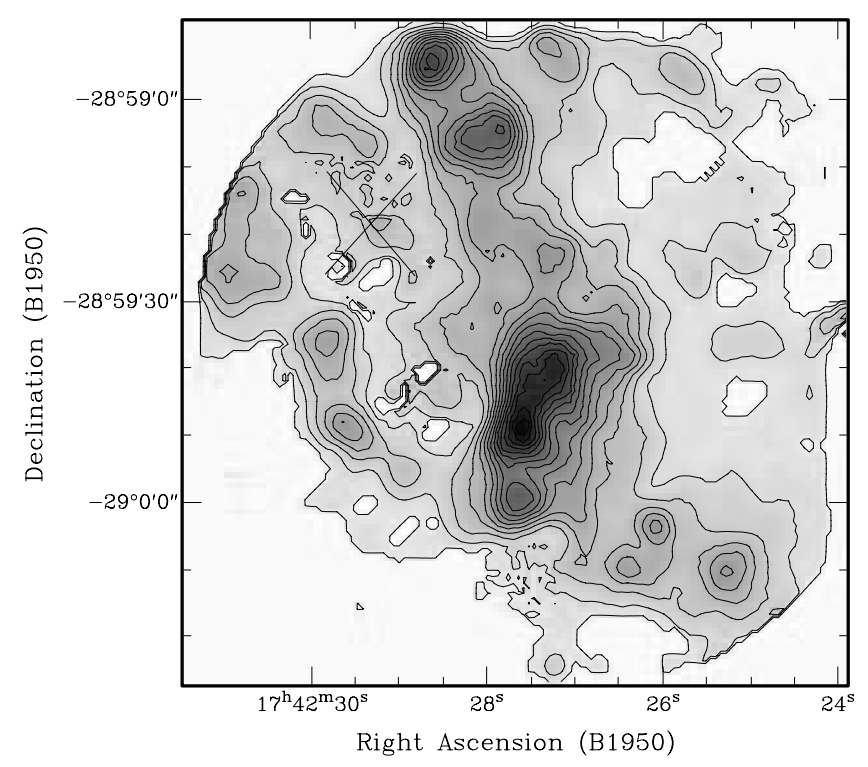

FIG. $6 a$

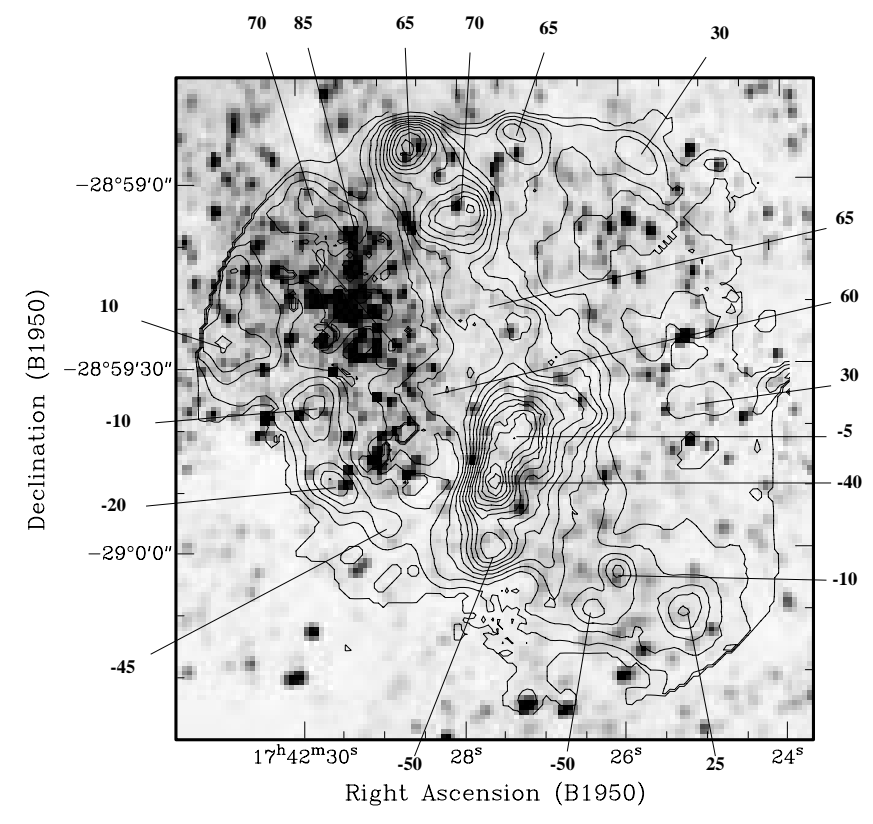

FIG. $6 b$

FIG. 6.-Same as Fig. 5, but for UNSWIRF field 2 of Fig. 1, the southern portion of the CND

observed fluxes in the [O I] $63 \mu \mathrm{m},\left[\mathrm{C}_{\mathrm{II}}\right] 158 \mu \mathrm{m}$, and [Si II] $35 \mu \mathrm{m}$ far-IR lines are consistent with a density of $\sim 10^{5}$ $\mathrm{cm}^{-3}$ and covering fraction $\sim 1$ (Burton et al. 1990; Wolfire, Tielens, \& Hollenbach 1990), consistent with UV heating of the envelopes of the dense cores seen in $\mathrm{HCN}$ (Jackson et al. 1993). In equilibrium, the UV-heated envelopes would produce a flux of only $\sim 10^{-5} \mathrm{erg} \mathrm{cm}^{-2}$ $\mathrm{s}^{-1} \mathrm{sr}^{-1}$. The $\mathrm{H}_{2}$ emission from the envelopes is increased substantially if their exposure to the UV flux varies on a timescale $\lesssim 300$ yr (Goldshmidt \& Sternberg 1995; Hollenbach \& Natta 1995). In this case, the 1-0 S(1) line intensity is $\sim 10^{-3} \mathrm{erg} \mathrm{cm}^{-2} \mathrm{~s}^{-1} \mathrm{sr}^{-1}$, the $2-1 / 1-0 S(1)$ ratio is $\sim 0.1$, and the far-IR line intensities are still matched because they reach equilibrium on a much shorter timescale (Hollenbach $\&$ Natta 1995). The only plausible source of variation that we can think of on this timescale is the shadowing by other clumps that would occur if $f_{A} \sim 1$ and the clumps have size $\sim 0.01 \mathrm{pc}$, assuming that the clump-clump velocity dispersion is $\sim 30 \mathrm{~km} \mathrm{~s}^{-1}$. A similar shadowing effect has been postulated to produce intense $\mathrm{C}_{\mathrm{I}}$ fine-structure emission from dense PDRs in molecular clouds (Störzer, Stutzki, \& Sternberg 1997).

The emission could also arise from gas heated by the dissipation of the $\approx 30 \mathrm{~km} \mathrm{~s}^{-1}$ velocity dispersion in the ring (e.g., Genzel 1989; Jackson et al. 1993), either by internal shock waves in a homogeneous but turbulent medium, or by collisions in a clumpy medium. The strength of the $\mathrm{H}_{2}$ line emission from clump collisions can be estimated as follows. Characterize the clumps by radius $r$, number density $n=n_{6} 10^{6} \mathrm{~cm}^{-3}$, and clump-to-clump velocity dispersion $v=v_{30}\left(30 \mathrm{~km} \mathrm{~s}^{-1}\right)$. In a collision, assume that a shock of area $\pi r^{2}$ and speed $v$ is driven into each cloud, and that a fraction $\epsilon$ of the mechanical energy flux $\frac{1}{2} \rho v^{3}$ incident on each shock is converted into emission in the 1-0 S(1) line of $\mathrm{H}_{2}$. Using the collision cross section, $\sigma \sim \pi(2 r)^{2}$, and the duration of a collision, $\sim 2 r / v$, then the fraction of clumps that are being shocked at any given time is $\approx$ $n_{\mathrm{cl}} \sigma v(2 r / v)=8 \pi r^{3} n_{\mathrm{cl}}=6 f_{V}$, where $n_{\mathrm{cl}}$ is the number of clumps per unit volume and $f_{V}$ is the volume-filling fraction of the clumps. The column density along the $\approx 1 \mathrm{pc}$ intersection of the line of sight with the lobes of the circumnuclear ring inferred from far-IR emission from dust grains is $\approx 10^{22} \mathrm{~cm}^{-2}$ (Latvakoski et al. 1999). This implies $f_{V} \approx$ $0.0032 / n_{6}$, and the line intensity is

$$
\begin{aligned}
I & =\frac{\epsilon \rho v^{3}}{8 \pi} 6 f_{V} f_{A} \\
& \approx 1.0 \times 10^{-3} \epsilon_{0.02} f_{A} v_{30}^{3} \mathrm{erg} \mathrm{cm}{ }^{-2} \mathrm{~s}^{-1} \mathrm{sr}^{-1},
\end{aligned}
$$

where $f_{A}$ is the area covering fraction of the clumps and $\epsilon=0.02 \epsilon_{0.02}$. Here $\epsilon$ is an initially increasing function of shock speed and density, dropping sharply for shock speeds in excess of $40-50 \mathrm{~km} \mathrm{~s}^{-1}$ when shocks become J-type (Hollenbach \& McKee 1989), and decreasing at densities $\gtrsim 10^{8} \mathrm{~cm}^{-3}$ because of collisional deexcitation of $\mathrm{H}_{2}$ (Kaufman \& Neufeld 1996b). The maximum value, $\sim 0.02$, is obtained for shock speeds between $\sim 30$ and $\sim 45 \mathrm{~km} \mathrm{~s}^{-1}$ and $n \sim 10^{5}-10^{7} \mathrm{~cm}^{-3}$ (Kaufman \& Neufeld 1996a). Thus, we conclude that clump collisions are capable of generating the observed intensity provided that $f_{A} \gtrsim 1$ and $n \gtrsim 10^{5}$ $\mathrm{cm}^{-3}$.

Both the time-dependent UV irradiation and clump collision models produce intensities that are roughly densityindependent, which may explain the uniformity of the $\mathrm{H}_{2}$ emission around the ring. Both scenarios also explain why the large-scale distribution of $\mathrm{H}_{2}$ emission from the ring traces the HCN $J=1-0$ emission reasonably well. The lack of obvious small-scale fluctuations in the $\mathrm{H}_{2}$ emission implies that several UV-irradiated clumps or colliding clumps must be present per square arcsecond. In the colliding clump scenario, this requires that the clump density $\gtrsim 10^{6} \mathrm{~cm}^{-3}$.

An additional process that could complicate the interpretation of $\mathrm{H}_{2}$ emission from the ring is its physical interaction with Sgr A East evidenced by $\mathrm{OH}(1720 \mathrm{MHz})$ masers. The new class of $\mathrm{OH}(1720 \mathrm{MHz})$ masers, which are also called "supernova masers," are very rare, and in all 20 observed sources that have been reported, the masers are 
physically associated with supernova remnants (Frail et al. 1996; Green et al. 1997; Koralesky et al. 1998; Yusef-Zadeh et al. 1999a). The most obvious SNR candidate associated with $\mathrm{OH}(1720 \mathrm{MHz})$ masers in the Galactic center is the nonthermal Sgr A East SNR driving a shock into the CND. Recent analysis of the $1720 \mathrm{MHz}$ maser observations carried out in 1986 shows a $-132 \mathrm{~km} \mathrm{~s}^{-1} \mathrm{OH}(1720 \mathrm{MHz})$ maser associated with the south lobe (Yusef-Zadeh et al. 2001). These high-velocity masers give compelling evidence that the masers of the CND are produced by the expansion of Sgr A East and that the $\mathrm{H}_{2}$ molecular emission in the CND is in part shock-excited externally by Sgr A East. In addition, the highly blue- and redshifted $\mathrm{OH}(1720 \mathrm{MHz})$ maser features correspond to the systemic velocity of the molecular gas in the CND. This is because the path of maximum amplification for inversion of the $1720 \mathrm{MHz} \mathrm{OH}$ maser is formed when the acceleration produced by the shock is transverse to the line of sight (Frail et al. 1994). This implies that the rotational velocity of the circumnuclear ring is about $130 \mathrm{~km} \mathrm{~s}^{-1}$.

\subsection{The $\mathrm{H}_{2}$ Linear Filament}

The width of the linear filament from the NICMOS observations is $\lesssim 1 " .3$, and the $\mathrm{H}_{2} 1-0 S(1)$ line intensity is $\approx 1 \times 10^{-4} \mathrm{erg} \mathrm{cm}^{-2} \mathrm{~s}^{-1} \mathrm{sr}^{-1}$. Several arguments imply that the filament is shock-heated gas rather than from a PDR. Shock models can easily produce the observed intensity and the $\mathrm{H}_{2}$ emission velocity peaks at $50-75 \mathrm{~km} \mathrm{~s}^{-1}$ (see Fig. 5b), which suggests that it is associated with $\mathrm{OH}$ $(1720 \mathrm{MHz})$ maser $\mathrm{C}$ at $43 \mathrm{~km} \mathrm{~s}^{-1}$. PDR models, on the other hand, require $G_{0} \sim 10^{6}, n_{\mathrm{H}} \gtrsim 10^{6} \mathrm{~cm}^{-3}$, and a high inclination to the line of sight (e.g., Burton et al. 1990). The $\mathrm{H}_{2}$ emission in a PDR arises in a layer of thickness corresponding to $A_{V} \approx 1$, which would be $\lesssim 2 \times 10^{15} \mathrm{~cm}$, corresponding to an angular scale $\lesssim 00^{\prime \prime} 02$. Further, there is no evidence for the associated ionization front as seen both in high-frequency radio continuum and $\mathrm{Br} \gamma$ line images. Interestingly, the $\mathrm{H}_{2}$ filament lies along the western edge of the Sgr A East shell (Yusef-Zadeh et al. 1999c), which is known to be nonthermal. Figure 7 shows the distribution of $\mathrm{H}_{2}$ line

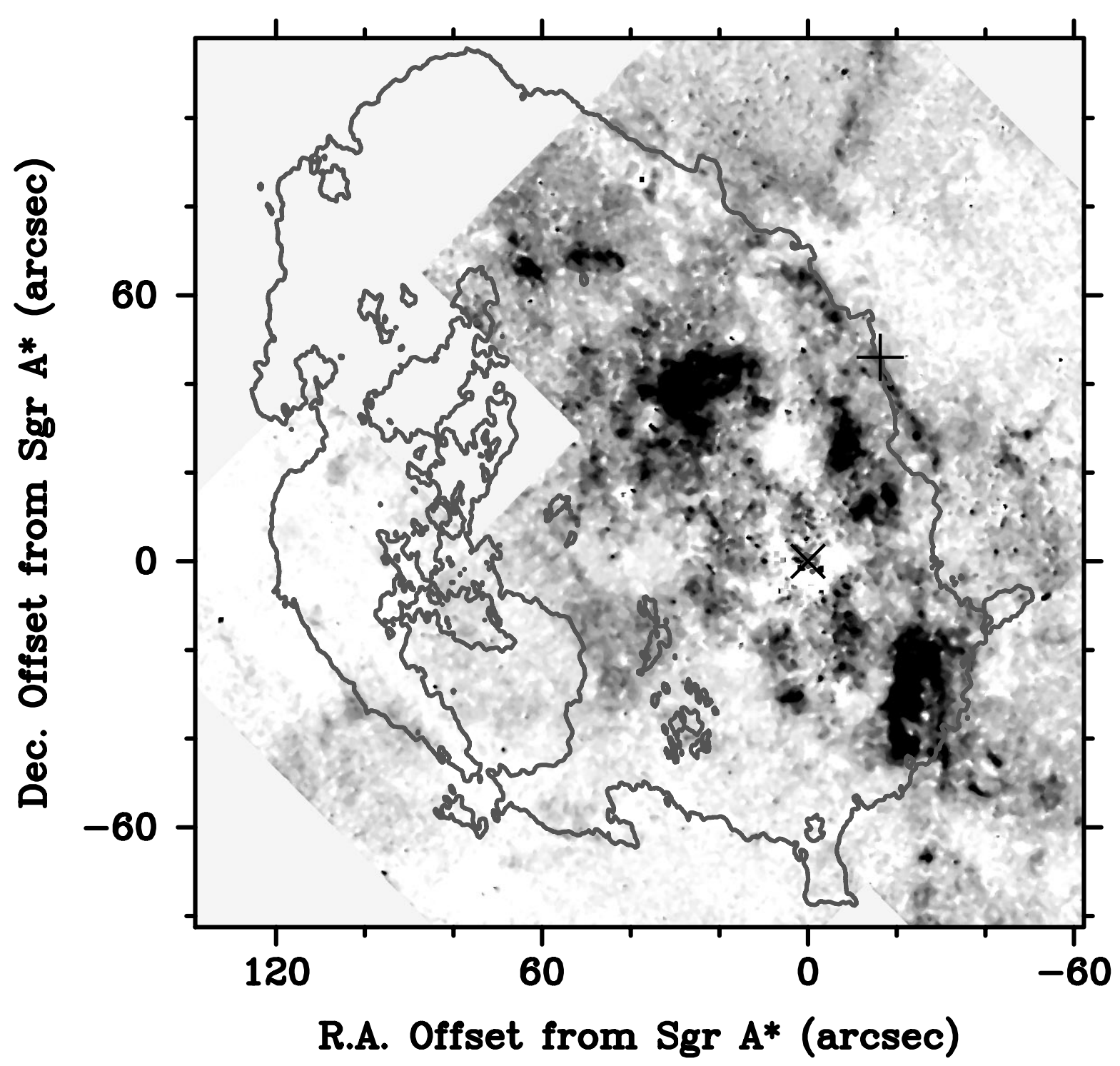

FIG. 7.- Contour of $20 \mathrm{~cm}$ continuum emission outlining the Sgr A East shell, shown at $0.75 \mathrm{mJy}^{\text {pixel }}{ }^{-1}$, pixel scale 0.5 and resolution 3 ". $1 \times 1$ 1"6, superimposed on the gray-scale continuum-subtracted NICMOS image of the $2.12 \mu \mathrm{m} \mathrm{H}_{2} 1-0 \mathrm{~S}(1)$ line emission from the CND and Sgr A East. The plus sign shows the position of maser source C from Yusef-Zadeh et al. (1996), and the cross shows the position of Sgr A*. 
emission based on NICMOS observations superimposed on a contour of $20 \mathrm{~cm}$ emission from the Sgr A East shell. The alignment of the western edge of the nonthermal Sgr A East shell, the linear $\mathrm{H}_{2}$ filament, and maser $\mathrm{C}$ is used as a compelling evidence that the $\mathrm{H}_{2}$ filament and the $\mathrm{OH}(1720$ $\mathrm{MHz}$ ) maser $\mathrm{C}$ are tracing shocked molecular gas produced by the expansion of Sgr A East into the western edge of the $50 \mathrm{~km} \mathrm{~s}^{-1}$ molecular cloud.

\section{4. $\mathrm{H}_{2}$ Emission and $\mathrm{OH}(1720 \mathrm{MHz})$ Masers}

Figure $2 a$ shows the large-scale view of $\mathrm{H}_{2}$ emission from NICMOS observations outlined by contours of $\mathrm{HCN}$ emission. A higher concentration of stars to the northeast is likely to be due to the Sgr A East $50 \mathrm{~km} \mathrm{~s}^{-1}$ cloud absorbing the background stellar continuum. Figure 2 shows a number of $\mathrm{H}_{2}$ emitting clouds beyond the contours of $\mathrm{HCN}$ emission. We describe below individual sources of $\mathrm{H}_{2}$ emission.

The region near $\alpha=17^{\mathrm{h}} 42^{\mathrm{m}} 32^{\mathrm{s}}, \delta=-29^{\circ} 00^{\prime} 30^{\prime \prime}$ to the southeast of Figure $2 b$ is of particular interest because a number of $\mathrm{OH}(1720 \mathrm{MHz})$ masers have been detected there (Yusef-Zadeh et al. 1996). The locations of the OH (1720 $\mathrm{MHz}$ ) maser features A and D-G (velocities of + 50-60 km $\mathrm{s}^{-1}$ ) within the M-0.02-0.07 (“ $50 \mathrm{~km} \mathrm{~s}^{-1}$ ") cloud are indicated in this figure. Spectroscopy toward these sources showed evidence of weak $\mathrm{H}_{2}$ emission lying close to the position of these masers (Wardle, Yusef-Zadeh, \& Geballe 1999). Figure 8 (F3 in Fig. 1) shows the integrated $\mathrm{H}_{2} 1-0$ $S(1)$ emission toward these masers. Similarly, Figure 9 shows the $\mathrm{H}_{2}$ distribution in an overlapping pointing ( $\mathrm{F} 4$ in Fig. 1).

The masers in Figures 8 and 9 appear to be aligned along an elongated nonthermal continuum feature at the southeastern boundary of Sgr A East at $20 \mathrm{~cm}$ (Yusef-Zadeh \& Morris 1987; Yusef-Zadeh et al. 1996). The $\mathrm{H}_{2}$ gas that appears to be associated with masers A-E (those labeled 66 and $57 \mathrm{~km} \mathrm{~s}^{-1}$ ) has a flux density of $5-10 \times 10^{-16} \mathrm{erg} \mathrm{s}^{-1}$ $\mathrm{cm}^{-2} \operatorname{arcsec}^{-2}$ and a mean velocity of about $95 \mathrm{~km} \mathrm{~s}^{-1}$. $\mathrm{NH}_{3}$ emission between -41 and $101 \mathrm{~km} \mathrm{~s}^{-1}$ (Coil \& Ho 1999) also lies adjacent to the maser sources, but is not seen

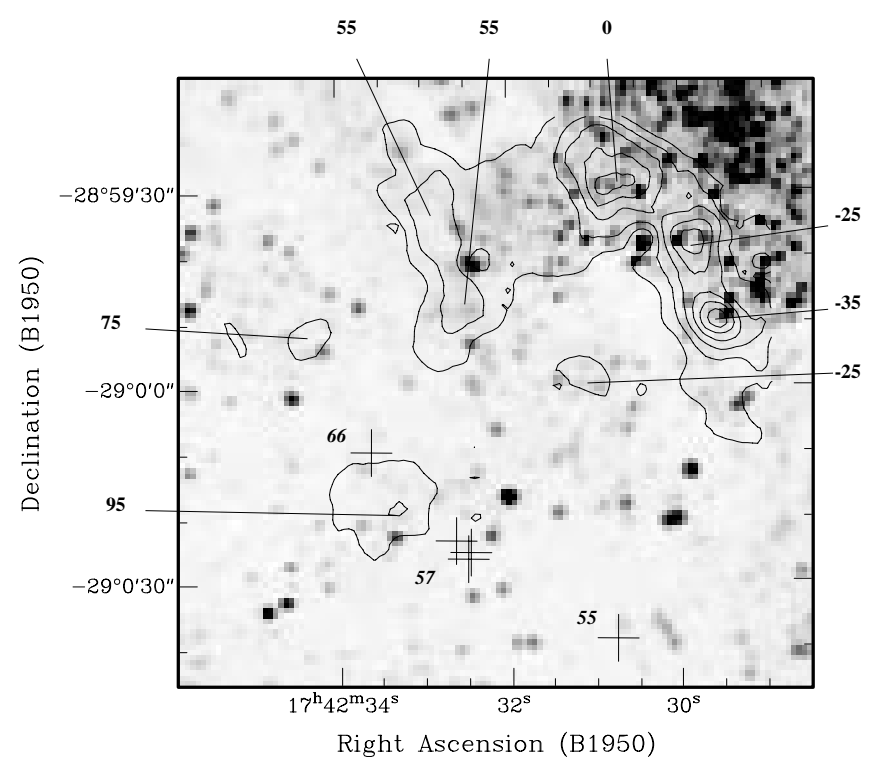

FIG. 8.-Same as Fig. $5 b$, but for UNSWIRF field 3 of Fig. 1, to the southeast of the CND.

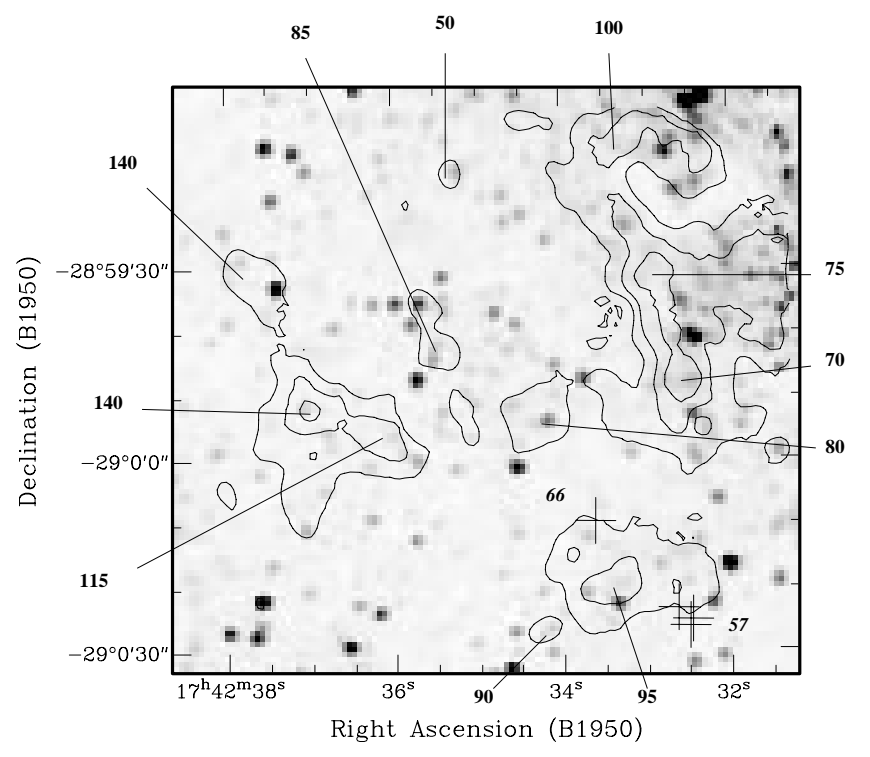

FIG. 9.-Same as Fig. $5 b$, but for UNSWIRF field 4 of Fig. 1, to the east of the CND.

associated with the north-south elongated $\mathrm{H}_{2}$ feature centered at $\alpha=17^{\mathrm{h}} 42^{\mathrm{m}} 32^{\mathrm{s}} .7, \delta=-28^{\circ} 59^{\prime} 45^{\prime \prime}$. The G OH $(1720$ $\mathrm{MHz}$ ) maser (labeled $55 \mathrm{~km} \mathrm{~s}^{-1}$ ) does not appear to have any detectable $\mathrm{H}_{2}$ counterpart. While the velocities of the $\mathrm{OH}$ masers and the corresponding $\mathrm{H}_{2}$ emission are not the same, differing by $\sim 30 \mathrm{~km} \mathrm{~s}^{-1}$ (as is the case for the $\mathrm{OH}$ masers in the northern lobe of the CND), they do support a physical association between them. The poor spatial correlation between $\mathrm{OH}$ masers and $\mathrm{NH}_{3}$ gas is probably explained by the restricted physical conditions under which $\mathrm{OH}(1720 \mathrm{MHz})$ masers can be formed (Lockett et al. 1999), as well as the fact that the velocities of $\mathrm{OH}(1720 \mathrm{MHz})$ masers trace the systemic motion of the molecular clouds. A number of high-velocity $\mathrm{H}_{2}$ features are also noted lying within and at the boundary of the Sgr A East shell (centered at $\alpha=17^{\mathrm{h}} 42^{\mathrm{m}} 32^{\mathrm{s}} \mathrm{7}, \quad \delta=-28^{\circ} 59^{\prime} 35^{\prime \prime}$ and $\alpha=17^{\mathrm{h}} 42^{\mathrm{m}} 37^{\mathrm{s}}$, $\delta=-28^{\circ} 59^{\prime} 50^{\prime}$ ). These are best shown in Figure 9 and have line center velocities ranging between 85 and $140 \mathrm{~km} \mathrm{~s}^{-1}$.

Two prominent $\mathrm{H}_{2}$ clouds are also noted in projection against the interior of the Sgr A East shell and to the north of the north lobe near $\alpha=17^{\mathrm{h}} 42^{\mathrm{m}} 33^{\mathrm{s}}, \delta=-28^{\circ} 58^{\prime} 15^{\prime \prime}$. These features, which are noted in Figures $2 a, 2 b$, and 7, are located about $90^{\prime \prime}$ northeast of Sgr A* and are labeled as "outer $\mathrm{H}_{2}$ clumps" in Figure $2 b$. The $20 \mathrm{~cm}$ image of this field shows a nonthermal ridge of emission at this location. The morphology of this ridge with respect to the distribution of the CND was recently discussed (see the supplement figure of Yusef-Zadeh, Melia, \& Wardle 2000) as possible evidence for the physical interaction of the CND and Sgr A East. The velocity structure of these $\mathrm{H}_{2}$ clouds is unknown, lying outside the UNSWIRF fields; thus, their physical association with either Sgr A East or the CND is unclear.

\subsection{The Outer $\mathrm{H}_{2}$ Filament and Clumps}

Another new straight filamentary structure, which appears somewhat broader and weaker than the linear filament, is shown in Figures $2 a$ and $2 b$. The typical $\mathrm{H}_{2}$ flux uncorrected for extinction ranges between 0.7 and $1.7 \times 10^{-15} \mathrm{erg} \mathrm{s}^{-1} \mathrm{~cm}^{-2} \operatorname{arcsec}^{-2}$, This feature is oriented roughly in the north-south direction and extends well 


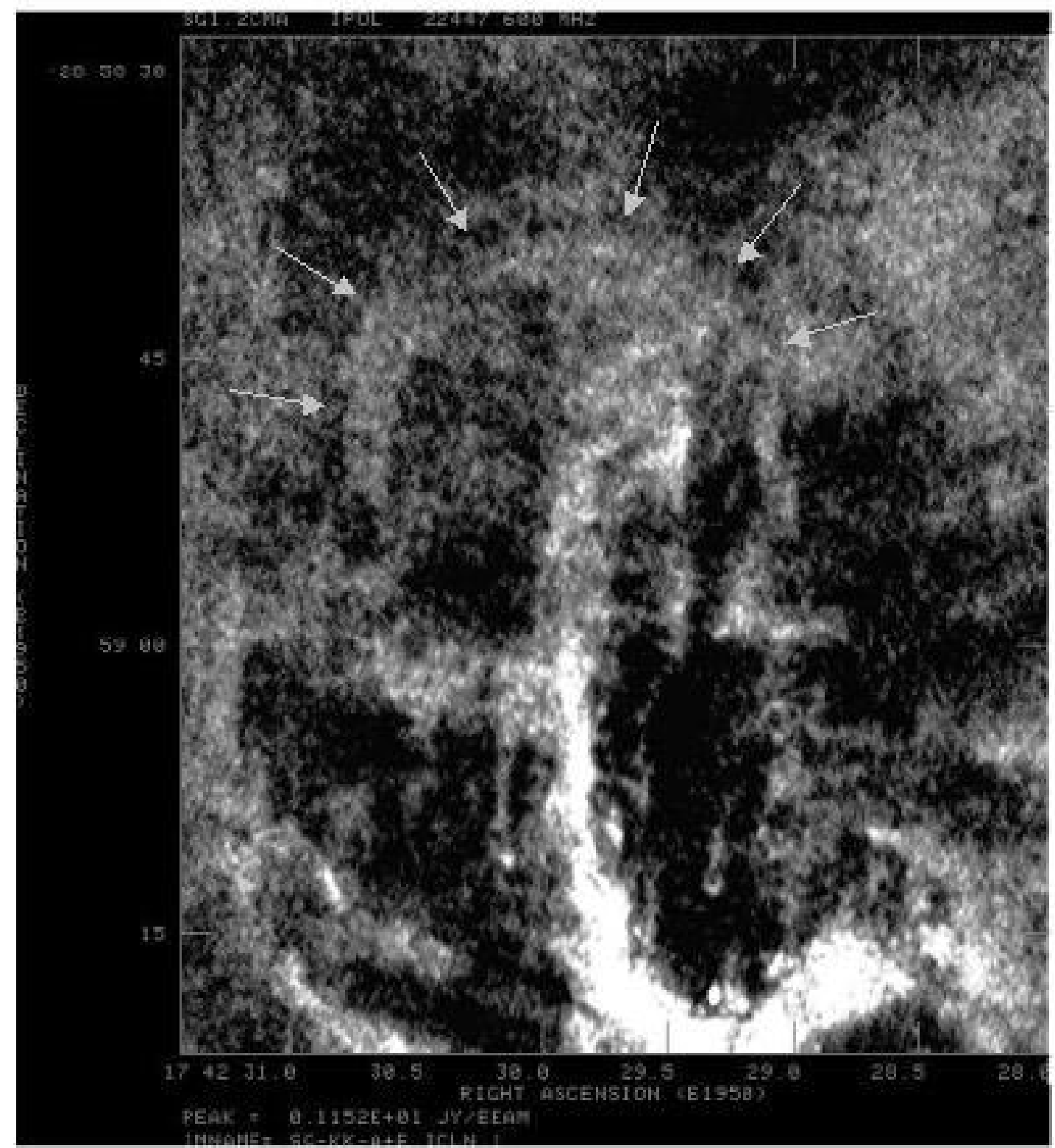

FIG. 10. - Gray-scale distribution of the ionized gas emitting in the continuum at $1.2 \mathrm{~cm}$, obtained with the VLA at a resolution of 0 " $3 \times 0$," 2 , showing the northern arm of Sgr A West together with a shell of weak ionized gas around its northern tip. In contrast to all other gray-scale images shown in this paper, this figure shows a positive gray-scale as the continuum emission is seen in white. The arrows are drawn to indicate the region of interest.

beyond the inner edge of the CND. This "outer filament" is seen to extend for at least $50^{\prime \prime}$ (and in fact may extend beyond the boundary of the NICMOS image). This straight but clumpy outer filament is of the order of $2^{\prime \prime}-3^{\prime \prime}$ in FWHM across and is coincident with a similarly elongated structure observed in the $34.8 \mu \mathrm{m}$ [Si II] line (Stolovy 1997). An enhancement in the [Si II]/dust continuum in this region also suggests an increase in the atomic silicon abundance due to grain destruction by shocks. In addition, a number of $\mathrm{H}_{2}$ clumps are seen throughout this region, two of which are located $50^{\prime \prime}$ east and $70^{\prime \prime}$ north of Sgr $\mathrm{A}^{*}$. The outer $\mathrm{H}_{2}$ clumps to the northeast of the northern lobe appear to be located between the inner edge of the nonthermal shell of Sgr A East and the outer edge of the CND.

\subsection{Extinction Clouds}

The prominent $\mathrm{H}_{2}$ filament as well as the north lobe and the western edge of the CND are clearly visible, in black, in
Figure $2 b$. A number of extinction clouds are apparent throughout the image (evident in white in the figure). Areas of high extinction are apparent in the continuum image (Fig. 1) as regions in white, marking a relative absence of stars, and in the $\mathrm{H}_{2}$ line image (Fig $2 b$, also in white) as regions where the residuals from imperfect continuum subtraction are least, a result of there being fewer stars there in the first place. Because the stellar flux is weak in its surface brightness at both the 2.12 and $2.15 \mu \mathrm{m}$ images, the areas of high extinction show up flatly near zero in the $\mathrm{H}_{2}$ image, which appears as white patches in the reverse gray scale. We discuss below the association of two prominent extinction clouds with Sgr A East and Sgr A West.

\subsubsection{North Arm Extinction Cloud}

An extinction feature in the NICMOS $\mathrm{H}_{2}$ image, with an angular size of about $20^{\prime \prime}$, coincides with the gap in the CND near $\alpha=17^{\mathrm{h}} 42^{\mathrm{m}} 30^{\mathrm{s}}, \delta=-28^{\circ} 58^{\prime} 50^{\prime \prime}$, or about $30^{\prime \prime}$ 
north of Sgr A* (see Figs. $2 a, 2 b$, and 7). Several factors suggest that this cloud is associated with the northern arm. The northernmost tip of the north arm in the $6 \mathrm{~cm}$ data, near IRS 8, as seen in Figure 3, coincides with the cloud. The kinematics of ionized gas along the north arm show multiple noncircular velocity components at $\alpha=$ $17^{\mathrm{h}} 42^{\mathrm{m}} 29^{\mathrm{s}} .8, \delta=-28^{\circ} 59^{\prime} 00^{\prime \prime}$, where the north arm bends in an otherwise continuous velocity distribution (Serabyn \& Lacy 1985). Stolovy, Scoville, \& Yusef-Zadeh (2001) have recently measured the extinction toward this cloud at subarcsecond resolution to be at least $A_{v} \sim 60 \mathrm{mag}$. It is likely higher than this in the darkest parts beyond the sensitivity of the measurement. In addition, the northern half of the north arm also shows a sudden drop by a factor of 2 in its surface brightness at $6 \mathrm{~cm}$ and a dearth of [Fe II] $1.64 \mu \mathrm{m}$ emission (Yusef-Zadeh et al. 1999b) to the north of $\alpha=$ $17^{\mathrm{h}} 42^{\mathrm{m}} 29^{\mathrm{s}} .7, \delta=-28^{\circ} 59^{\prime} 00^{\prime \prime}$ - i.e., where the extinction feature is (Yusef-Zadeh \& Wardle 1993). However, the strongest evidence that the extinction cloud lies within the CND comes from the $\lambda 1.2 \mathrm{~cm}$ VLA continuum image shown in Figure 10. This shows the north Arm of Sgr A West and a faint semicircular shell of ionized gas surrounding the northern tip of the north arm. Similarly, a radiograph of this region (see Fig. 4 of Yusef-Zadeh \& Morris 1987) reveals what appears to be a limb-brightened hole in the distribution of continuum emission at this location. The morphology in Figure 10 is suggestive of a neutral cloud surrounded by ionized gas (Yusef-Zadeh, Zhao, \& Goss 1995). The ionized shell suggests that the cloud is photoionized externally and is associated with the northern tip of the north arm. Indeed, this feature coincides with the outer rim of the extinction cloud seen with NICMOS at $2.12 \mu \mathrm{m}$. Its surface brightness at $1.2 \mathrm{~cm}, \sim 0.2 \mathrm{mJy}$ within a $0.3 \times 0.2$ beam, is at the level expected for the ionizing radiation field within the cavity. This cloud is possibly the single cloud from which the north arm originated before falling on a circular orbit toward Sgr A*. Earlier kinematic studies of $12.8 \mu \mathrm{m}[\mathrm{Ne}$ II] line emission by Serabyn \& Lacy (1985) predicted such a cloud would be colliding with the CND. The cloud has also been detected in an $\mathrm{H}$ I absorption study of this region, where it appears as a feature at $130 \mathrm{~km} \mathrm{~s}^{-1}$ (Plante, Lo, \& Crutcher 1995). The position, strength and direction of $\mathrm{H}$ I Zeeman splitting measurements at +130 $\mathrm{km} \mathrm{s}^{-1}$ agree with the Zeeman study of the $1720 \mathrm{MHz} \mathrm{OH}$ masers at the same location (Plante et al. 1995; YusefZadeh et al. 1996).

\subsubsection{The Extended Extinction Cloud}

Another prominent and extended extinction feature is located to the northwest of the linear $\mathrm{H}_{2}$ filament, extending northeast-southwest from around $\alpha=17^{\mathrm{h}} 42^{\mathrm{m}} 27^{\mathrm{s}}$, $\delta=-28^{\circ} 58^{\prime} 15^{\prime \prime}$ or about $70^{\prime \prime}$ northwest of $\mathrm{Sgr} \mathrm{A}^{*}$ in Figures $2 a, 2 b$, and 7 . The boundaries of this feature are not well defined, but it appears to "wrap around" the linear filament and continue southward, connecting to the northern arm extinction cloud described in the previous subsection. The contour of $20 \mathrm{~cm}$ emission in Figure 7 runs parallel to the inner edge of this extinction feature.
The eastern side of Sgr A East is known to be interacting with the $50 \mathrm{~km} \mathrm{~s}^{-1}$ cloud (e.g., Mezger et al. 1989; Zylka et al. 1990; Serabyn et al. 1992; Yusef-Zadeh et al. 1996; Coil $\&$ Ho 1999). However, the interaction of the western half of Sgr A East has not been examined in detail before. The extended extinction cloud discussed here may also be associated with the well-known $50 \mathrm{~km} \mathrm{~s}^{-1}$ cloud, assuming that the $\mathrm{OH}(1720 \mathrm{MHz})$ maser at $43 \mathrm{~km} \mathrm{~s}^{-1}$ represents the systemic motion of the cloud at its interaction site with Sgr A East.

A number of weakly emitting radio continuum features, known as the streamers, have also been recognized running perpendicular to the CND (Yusef-Zadeh \& Morris 1987; Yusef-Zadeh \& Wardle 1993). H110 $\alpha$ observations also indicate gas velocities up to $+144 \mathrm{~km} \mathrm{~s}^{-1}$ near $\alpha=17^{\mathrm{h}} 42^{\mathrm{m}} 28^{\mathrm{s}}, \delta=-28^{\circ} 58^{\prime} 15^{\prime \prime}$ (just to the northwest of the $\mathrm{H}_{2}$ filament). Along with the kinematics of the ionized gas near the $43 \mathrm{~km} \mathrm{~s}^{-1} \mathrm{OH}$ maser, these velocities are inconsistent with circular orbital motion about the Galactic nucleus. (Yusef-Zadeh et al. 1995). This suggests that these features in the ionized gas are also associated with the same molecular cloud producing the extinction feature at 2.12 $\mu \mathrm{m}$.

\section{CONCLUSIONS}

In summary, we have presented $\mathrm{H}_{2}$ 1-0 S(1) line observations of the circumnuclear ring and Sgr A East using NICMOS on the HST and UNSWIRF on the AAT. These high spatial and spectral resolution images are correlated with $\mathrm{OH}(1720 \mathrm{MHz})$ masers as well as $\mathrm{Br} \gamma$ line and radio continuum observations of the complex region of the Galactic center. In spite of the difficulty of detecting shocked molecular gas due to the large intrinsic line width of Galactic center molecular clouds, these observations show strong evidence of hot shocked $\mathrm{H}_{2}$ gas and cool postshock gas as traced by $\mathrm{OH}(1720 \mathrm{MHz})$ masers. The interaction of two dynamically coupled systems associated with Sgr A East and the cicumnuclear ring is discussed. This argument is based on the fact that in all 20 supernova masers that have been discovered in the Galaxy, OH (1720 $\mathrm{MHz}$ ) masers are clearly evident at the boundary of supernova remnants and molecular clouds where the interaction is taking place. In the Galactic center region, the expansion of the nonthermal shell of Sgr A East drives a shock into both the $50 \mathrm{~km} \mathrm{~s}^{-1}$ cloud and the circumnuclear ring and produces the observed $\mathrm{OH}(1720 \mathrm{MHz})$ masers. Finally, we have argued that the molecular $\mathrm{H}_{2}$ emission from the circumnuclear ring results from gas heated in the dissipation of the random motion of molecular clumps in the ring.

We thank Ylva Schuberth and Tony Travouillon for their help in reduction of the UNSWIRF data. We also thank D. Roberts for useful discussions. The research described in this paper was carried out in part by NASA grant GO07844 and the Jet Propulsion Laboratory, California Institute of Technology, under contract to NASA. 
Allen, D. A., Hyland, A. R., \& Hillier, D. J. 1990, MNRAS, 244, 706

Baganoff, F. K., et al. 2001, ApJ, in press

Burton, M., \& Allen, D.A. 1992, Proc. Astron. Soc. Australia, 10, 55 1993, in ASP Conf. Ser. 41, Astronomical Infrared Spectroscopy: Future Observational Directions, ed. S. Kwok (San Francisco: ASP), 289

Burton, M. G., Hollenbach, D. J., \& Tielens, A. G. G. M. 1990, ApJ, 365, 620

Coil, A., \& Ho, P. T. P. 1999, ApJ, 513, 752

Draine, B. T., Roberge, W. G., \& Dalgarno, A. 1983, ApJ, 264, 485

Eckart, A., \& Genzel, R. 1996, Nature, 383, 415

Frail, D. A., Goss, W. M., Reynoso, E. M., Green, A. J., \& Otrupcek, R. 1996, AJ, 111, 1651

Frail, D. A., Goss, W. M., \& Slysh, V. I. 1994, ApJ, 424, L111

Gatley, I., Beattie, D. H., Lee, T. J., Jones, T. J., \& Hyland, A. R. 1984 MNRAS, 210, 565

Gatley, I., Jones, T. J., Hyland, A. R., Wade, R., Geballe, T. R., \& Krisciunas, K. 1986, MNRAS, 222, 299

Geballe, T. R., Krisciunas, K., Bailey, J. A., \& Wade, R. 1991, ApJ, 370, L73

Genzel, R. 1989, in IAU Symp. 136, The Center of the Galaxy, ed. M. Morris (Dordrecht: Kluwer), 393

Genzel, R., Hollenbach, D., \& Townes, C. H. 1995, Rep. Prog. Phys., 57, 417

Ghez, A., Klein, B. L., Morris, M., \& Becklin, E. E. 1998, ApJ, 509, 678

Goldshmidt, O., \& Sternberg, A. 1995, ApJ, 439, 256

Green A. J., Frail, D. A., Goss, W. M., \& Otrupcek, R. 1997, AJ, 114, 2058

Güsten, R., Genzel, R., Wright, M. C. H., Jaffe, D. T., Stutzki, J., \& Harris, A. I. 1987, ApJ, 318, 124

Hall, D. N. B., Kleinmann, S. G., \& Scoville, N. Z. 1982, ApJ, 260, L53

Hollenbach, D. J., \& McKee, C. F. 1989, ApJ, 342, 306

Hollenbach, D., \& Natta, A. 1995, ApJ, 455, 133

Jackson, J. M., Geis, N., Genzel, R., Harris, A. I., Madden, S., Poglitsch, A., Stacey, G. J., \& Townes, C. H. 1993, ApJ, 402, 173

Kaufman, M. J., \& Neufeld, D. A. 1996a, ApJ, 456, 611 1996b, ApJ, 456, 250

Koralesky, B., Frail, D. A., Goss, W. M., Claussen, M. J., \& Green, A. J. 1998, AJ, 116, 1323

Krabbe, A., Genzel, R., Drapatz, S., \& Rotaciuc, V. 1991, ApJ, 382, L19

Kwan, J. 1977, ApJ, 216, 713

Latvakoski, H. M., Stacey, G. J., Gull, G. E., \& Hayward, T. L. 1999, ApJ, 511,761

Lockett, P., Gauthier, E., \& Elitzur, M. 1999, ApJ, 511, 235

Maeda, Y., et al. 2001, in press

Marshall, J., Lasenby, A. N. \& Harris, A. I. 1995, MNRAS, 277, 594
REFERENCES

Menten, K., Reod, M., Eckart, A., \& Genzel, R. 1997, ApJ, 475, L111

Mezger, P. G., Zylka, R., Salter, C. J., Wink, J. E., Chini, R., \& Kreysa, E. 1989, A\&A, 209, 337

Najarro, F., Krabbe, A., Genzel, R., Lutz, D., Kudritzki, R. P., \& Hillier, D. J. 1997, A\&A, 325, 700

Pak, S., Jaffe, D. T., \& Keller, L. D. 1996, ApJ, 457, L43

Plante, R. L., Lo, K. Y., \& Crutcher, R. M. 1995, ApJ, 445, L113

Ramsay-Howat, S., Mountain, M., \& Geballe, T. R. 1993, in ASP Conf Ser. 41, Astronomical Infrared Spectroscopy: Future Observational Directions, ed. S. Kwok (San Francisco: ASP), 3

Roberts, D. A., \& Goss, W. M. 1993, ApJS, 86, 133

Roberts, D. A., Yusef-Zadeh, F., \& Goss, W. M. 1996, ApJ, 459, 627

Ryder, S. D., Sun, Y-S., Ashley, M. C. B., Burton, M. G., Allen, L. E., \& Storey, J. W. V. 1998, MNRAS, 294, 338

Serabyn, E., \& Lacy, J. H. 1985, ApJ, 293, 445

Serabyn, E., Lacy, J. H., \& Achtermann, J. M. 1992, ApJ, 395, 166

Sternberg, A., \& Dalgarno, A. 1989, ApJ, 338, 197

Störzer, H., Stutzki, J., \& Sternberg, A. 1997, A\&A, 323, L13

Stolovy, S. R. 1997, Ph.D. thesis, Cornell Univ.

Stolovy, S. R., Scoville, N., \& Yusef-Zadeh, F. 2001, PASP, in press

Wardle, M. 1999, ApJ, 525, L101

Wardle, M., Yusef-Zadeh, F., \& Geballe, T. R. 1999, in ASP Conf. Ser. 186, The Central Parsecs, ed. H. Falcke, et al. (San Francisco: ASP), 432

Wolfire, M. G., Tielens, A. G. G. M., \& Hollenbach, D. 1990, ApJ, 358, 116

Wright, M. C. H., Genzel, R., Güsten, R., \& Jaffe, D. T. 1987, in The Galactic Center, ed. D. C. Backer (New York: AIP), 133

Yusef-Zadeh, F., Goss, W. M., Roberts, D. A., Robinson, B., \& Frail, D. 1999a, ApJ, 512, 230

Yusef-Zadeh, F., Melia, F., \& Wardle, M. 2000, Science, 287, 95

Yusef-Zadeh, F., \& Morris, M. 1987, ApJ, 322, 721

Yusef-Zadeh, F., Roberts, D. A., Bower, G., Wardle, M., \& Goss, W. M. 2001, in IAU Symp. 206, Cosmic Masers, ed. V. Migenes (New York: AIP), in press

Yusef-Zadeh, F., Roberts, D. A., Goss, W. M., Frail, D., \& Green, A. 1996, ApJ, 466, L25

Yusef-Zadeh, F., Roberts, D. A., Goss, W. M., Frail, D., \& Green, A. 1999b, ApJ, 512, 230

Yusef-Zadeh, F., Stolovy, S., Wardle, M., Melia, F., Roberts, D., Kassim, N., \& Lazio J. 1999c, in ASP Conf. Ser. 186, The Central Parsecs, ed. H. Falcke, et al. (San Francisco: ASP), 197

Yusef-Zadeh, F., \& Wardle, M. 1993, ApJ, 405, 584

Yusef-Zadeh, F., Zhao, J. H., \& Goss, W. M. 1995, ApJ, 442, 646

Zylka, R., Mezger, P. G., \& Wink, J. E. 1990, A\&A, 234, 133 